This is the accepted manuscript made available via CHORUS. The article has been published as:

Charge-neutral disorder and polytypes in heterovalent wurtzite-based ternary semiconductors: The importance of the octet rule

Paul C. Quayle, Eric W. Blanton, Atchara Punya, Grant T. Junno, Keliang He, Lu Han, Hongping Zhao, Jie Shan, Walter R. L. Lambrecht, and Kathleen Kash

Phys. Rev. B 91, 205207 - Published 18 May 2015

DOI: 10.1103/PhysRevB.91.205207 


\title{
Charge-neutral disorder and polytypes in heterovalent wurtzite-based ternary semiconductors: the importance of the octet rule
}

\author{
Paul C. Quayle ${ }^{1}$, Eric W. Blanton ${ }^{1}$, Atchara Punya ${ }^{1,3}$, Grant T. Junno ${ }^{1}$, Keliang $\mathrm{He}^{1}$, \\ Lu Han ${ }^{2}$, Hongping Zhao ${ }^{2}$, Jie Shan ${ }^{1}$, Walter R. L. Lambrecht ${ }^{1}$, and Kathleen Kash ${ }^{1}$ \\ ${ }^{1}$ Department of Physics, Case Western Reserve University, Cleveland, OH 44106-7079 \\ 2 Department of Electrical Engineering and Computer Science, \\ Case Western Reserve University, Cleveland, OH 44106-7071 and \\ 3 Department of Physics and Materials Science, Chiang Mai University, \\ 239 Huay Kaew Road, Muang, Chiang Mai 50200 Thailand
}

\begin{abstract}
We investigate lattice ordering phenomena for the heterovalent ternaries that are based on the wurtzite lattice, under the constraint that the octet rule be preserved. We show that, with the single exception of a highly symmetric twinned structure, all allowed lattice orderings can be described by a pseudospin model corresponding to the two different stackings of $A B A B$ rows of atoms in the basal plane that occur in the $P n a 2_{1}$ and $P m c 2_{1}$ crystal structures. First-principles calculations show that the difference in the energies of formation between these two structures is $13 \pm 3 \mathrm{meV} / \mathrm{fu}$ (formula unit) for $\mathrm{ZnSnN}_{2}$ and is an order of magnitude larger for $\mathrm{ZnGeN}_{2}$, and that for both materials the Pm31 structure, which contains only octet-rule-violating tetrahedra, has a significantly higher energy of formation and a signficantly lower band gap. We predict almost random stacking and wurtzite-like x-ray diffraction spectra in the case of $\mathrm{ZnSnN}_{2}$, consistent with reported measurements. The octet-rule-preserving model of disorder proposed here predicts a band gap that for $\mathrm{ZnSnN}_{2}$ is relatively insensitive to ordering, in contrast to the prevailing model, which invokes the random placement of atoms on the cation sublattice. The violations of the octet rule in the latter model lead to significant narrowing of the band gap. The Raman and photoluminescence spectra of $\mathrm{ZnSnN}_{2}$ are interpreted in light of the ordering model developed here. The observation that $\mathrm{ZnGeN}_{2}$ orders in the Pna2 $2_{1}$ structure under appropriate growth conditions is consistent with the larger difference in the energies of formation of the $P n a 2_{1}$ and $P m c 2_{1}$ structures for this material. The ordering model presented here has important implications for the optical, electronic and lattice properties of all wurtzite-based heterovalent ternaries.
\end{abstract}

PACS numbers: 61.50.-f, 78.20.-3

\section{INTRODUCTION}

The heterovalent ternaries are a large family of materials that are close cousins of the binary zincblende and wurtzite semiconductors. Conceptually, either the cation or the anion lattice of the binary material is replaced by two ordered sublattices composed of equal proportions of two atomic species such that the average ratio of valence electrons to atoms equals four, and the bonding remains tetrahedral. Ideally, the ordering of the atoms on the two sublattices of the heterovalent compound is constrained to satisfy local charge neutrality, a constraint referred to in general as the octet rule. The II-IV- $\mathrm{V}_{2}$ ternaries and their alloys thus provide an interesting alternative to the isovalent III-V alloys for band structure engineering of semiconductors.

While alloys of the binaries usually have a random distribution of cations, the pure ternaries tend to form ordered compounds. The II-IV- $\mathrm{V}_{2}$ and I-III$\mathrm{VI}_{2}$ compounds based on the zincblende lattice are well known to order in the chalcopyrite structure ${ }^{1,2}$ and have received significant attention for their nonlinear optical properties and photovoltaic applications. For example, $\mathrm{Cu}(\mathrm{In}, \mathrm{Ga})(\mathrm{S}, \mathrm{Se})_{2}$ thin films are widely used in photovoltaics ${ }^{3-5}$ and $\mathrm{ZnGeP}_{2}, \mathrm{CdGeAs}_{2}$ and $\mathrm{AgGa}(\mathrm{Se}, \mathrm{Te})_{2}$ single crystals are used as frequency dou- blers and parametric oscillators in nonlinear optical applications. ${ }^{6-8}$

Order-disorder transitions of these chalcopyrite materials have been reported, with the disordered state apparently exhibiting the binary, parent zincblende structure in x-ray diffraction spectra. ${ }^{1,2}$ The accepted model, first proposed by Buerger in 1934, is that the cations undergo entropy-driven randomization of their positions in the two cation sublattices. ${ }^{9}$ This randomization is necessarily accompanied by many local violations of the octet rule, although the rule is observed on average, for stoichiometric material. Thus, some tetrahedra have, for example, one $A$ and three $B$ cations or vice versa rather than two of each. Wei et al. ${ }^{10}$ used a generalized one-dimensional Ising model, similar to the model developed here for the wurtzite-based ternaries, to investigate the band structure and stability of polytypes of the zincblende-based ternary materials obeying the octet rule.

Recently, the more ionic, wurtzite-based ternary nitrides have generated renewed interest. ${ }^{11}$ Some are predicted to have important potential for several applications in optoelectronics, from solid-state lighting ${ }^{12}$ to photovoltaics. ${ }^{13}$ The synthesis of $\mathrm{ZnSnN}_{2}$ in particular has fueled interest in its use for solar photovoltaics, especially because this material is composed solely of earthabundant elements. ${ }^{14-21}$ As is the case for the zincblendebased ternaries, the type of ordering and the possibility of 


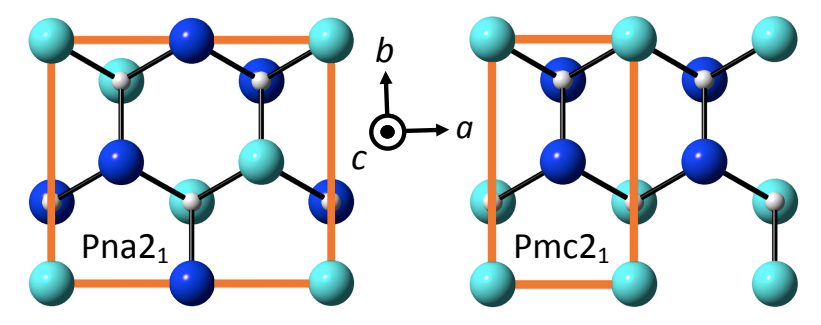

FIG. 1: (Color online) Projection of the $\mathrm{ABC}_{2}$ Pna $2_{1}$ and $P m c 2_{1}$ crystal structures on the c-plane, with principal axes as indicated and the unit cells outlined. Large spheres are the cations, small spheres are the anions.

order-disorder transitions in the wurtzite-based ternaries are of fundamental as well as practical interest.

For $\mathrm{ZnGeN}_{2}$ and $\mathrm{ZnSnN}_{2}$ the parent binary materials are the III-nitrides, and the parent binary phase is wurtzite. $\mathrm{ZnGeN}_{2}$ was first synthesized in 1970, and its crystal structure was reported initially to be monoclinic. ${ }^{22,23}$ Subsequent reports variously reported the structure to be monoclinic, ${ }^{22}$ orthorhombic $^{24}$ or hexagonal. The currently accepted crystal structure is the sixteen-atom orthorhombic unit cell pictured in Fig. 1 on the left. The relation between this superstructure, with the Pna $2_{1}$ space group, and the wurtzite unit cell in the ideal case (that is, with no relaxations of the wurtzite atomic positions) is $\mathbf{a}_{o}=2 \mathbf{a}_{w 1}, \mathbf{b}_{o}=\mathbf{a}_{w 1}+2 \mathbf{a}_{w 2}$, $\mathbf{c}_{o}=\mathbf{c}_{w}$, where the subscripts $w$ denote the wurtzite lattice vectors and the subscripts $o$ denote the orthorhombic lattice vectors. The band gap calculated by the quasiparticle self-consistent $G W(\mathrm{QS} G W) \operatorname{method}^{25,26}$ is in close agreement with the experimental value obtained from near-band-gap luminescence ${ }^{27}$ of $3.40 \pm 0.01 \mathrm{eV}$.

Comparison of the calculated Raman frequencies, intensities and polarization selection rules for $\mathrm{ZnGeN}_{2}$ yielded close agreement with measurements, ${ }^{28}$ but the experimental spectra intriguingly included additional features that were identified with peaks in the phonon density of states, indicating that some form of disorder is present in the samples. It has been demonstrated that the x-ray diffraction spectra could be varied from wurtzitic to the fully-ordered orthorhombic Pna $2_{1}$ structure through the choice of growth conditions. ${ }^{29}$

Reported calculations of the band gap of $\mathrm{ZnSnN}_{2}$ in the $P n a 2_{1}$ structure differ widely, from 1.4 to $2 \mathrm{eV} .{ }^{14,17,26}$ At least some of these differences may simply be due to differences in the lattice parameters and computational methods used. (Different hybrid functionals ${ }^{30-32}$ were used in Refs. 14 and 17 and QSGW was used in Ref. 26.) Synthesis of $\mathrm{ZnSnN}_{2}$ was first reported in 2012 by molecular beam epitaxy ${ }^{14}$, soon afterward by radio frequency $(\mathrm{RF})$ sputter deposition ${ }^{16,17}$ and by a vapor-liquid-solid method ${ }^{19}$, and more recently by direct current (DC) magnetron sputtering ${ }^{21}$. All four methods yielded wurtzitic x-ray diffraction spectra, implying considerable disorder on the cation sublattices that was presumed to involve many violations of the octet rule. It was suggested that controlling the degree of this disorder would allow the controlled variation of the band gap from 1 to $2 \mathrm{eV} .{ }^{15}$ However, measurement of the band gap by photoluminescence excitation spectroscopy ${ }^{19}$ of material that looked wurtzitic in x-ray diffraction gave a gap in close agreement with accurately predictive quasiparticle band structure calculations for perfectly ordered Pna $2_{1}$ crystals, using experimentally determined lattice parameters. $^{13,26}$ The main question we address in this paper is how to reconcile these seemingly contradictory observations.

A second possible octet-rule-preserving orthorhombic structure, with the $P m c 2_{1}$ space group and consisting of an 8-atom unit cell with vectors $\mathbf{a}_{o}=\mathbf{a}_{w 1}, \mathbf{b}_{o}=$ $\mathbf{a}_{w 1}+2 \mathbf{a}_{w 2}, \mathbf{c}_{o}=\mathbf{c}_{w}$, was recently proposed, ${ }^{17}$ and is depicted in Fig. 1 on the right. For $\mathrm{ZnSnN}_{2}$, the calculated energy of formation of the $P m c 2_{1}$ structure was reported to be equal to that of the $P n a 2_{1}$ structure to within the computational accuracy of $10 \mathrm{meV}$ per nitrogen atom. Observation of the $P m c 2_{1}$ structure, however, has not yet been reported.

In this paper, we propose that the lattice disorder in $\mathrm{ZnSnN}_{2}$ evident in the x-ray diffraction spectra results not from the random placement of cations on the group III sublattice, but from a more constrained type of disorder that preserves local charge neutrality. This proposal is based on first-principles calculations for the $P n a 2_{1}$ and $P m c 2_{1}$ structures, which obey the octet rule, and the Pm31 structure, which does not. The two structures that obey the octet rule have very close energies of formation and band gaps, while the structure violating the octet rule has a substantial cost in the energy of formation and a much lower band gap. Based on these calculations, we then investigate the possible orderings of cations on the wurtzite cation sublattice with the restriction that the octet rule must be obeyed. We demonstrate that all possible orderings under this constraint can be viewed as combinations of the row stackings in the basal plane occurring in $P n a 2_{1}$ and $P m c 2_{1}$. Regular arrangements of these stackings define a polytype ordering and, in the case of random stacking, a new type of disorder for these materials. Using this approach, we find that ordered $\mathrm{ZnSnN}_{2}$ is thermodynamically unfavorable. Furthermore, the band gap of $\mathrm{ZnSnN}_{2}$ should be relatively insensitive to this type of disorder. Thus, the $\mathrm{ZnSnN}_{2}$ paradox mentioned earlier is resolved. In the case of $\mathrm{ZnGeN}_{2}$ the lattice has been observed to range from ordered $P n a 2_{1}$ to disordered, depending on growth conditions. This situation is likely the result of kinetic rather than thermodynamic factors.

The ordering model developed here has broad implications for the optical, electronic and lattice properties of all wurtzite-based ternary materials, and possibly even calls for a re-examination of the question of ordering in the zincblende-based ternaries.

The remainder of the paper is organized as follows. First, we introduce the lattice ordering pseudospin model 
in Sec. II while relegating the details of the proofs to Appendix A. Next, we provide the details of the computational and experimental methods in Sec. III. Finally, new experimental and calculated results for $\mathrm{ZnSnN}_{2}$ and $\mathrm{ZnGeN}_{2}$ are presented in Sec. IV. A brief summary (Sec. V) concludes the paper.

\section{LATTICE ORDERING IN WURTZITE-BASED HETEROVALENT TERNARIES}

Based on first-principles results for the energies of formation, we focus on orderings of the cations that preserve the octet rule in every nearest-neighbor tetrahedron. In this section, we show that all of these can be described in terms of a layered pseudo-spin model.

We first illustrate that one can build octet-rulepreserving polytypes of the $P m c 2_{1}$ and $P n a 2_{1}$ unit cells (Figure 1). If the $P m c 2_{1}$ unit cell is rotated from its orientation in Fig. 1 by $120^{\circ}$, as shown in Fig. 2, the rows of cations have $A B A B$ periodicity, as do the rows of cations along the a-axis in the $P n a 2_{1}$ structure. Thus the $P n a 2_{1}$ and $P m c 2_{1}$ structures can be obtained by stacking such rows along the $\mathbf{b}$-axis.

We define the $s= \pm 1$ pseudospin layers as shown in Figs. 2(c) and (d). The dashed lines outline primitive cells of the $P m c 2_{1}$ structure. One can clearly see that the two layers are mirror images of each other for a vertical mirror plane. The $P n a 2_{1}$ structure results from $+1,-1$ stacking of these pseudospin layers along the $P n a 2_{1}$ baxis, and the $P m c 2_{1}$ structure results from $+1,+1$ or $-1,-1$ stacking. It follows that an infinite number of polytypes can be built from larger repeat units. In Appendix A we show rigorously that the only ternary crystal structures based on the wurtzite lattice that observe local charge neutrality are those described by sequences of the \pm 1 pseudospin layers, with the sole exception of the structure shown in Fig. 2(e). This unique structure is a six-fold twinning of the $P m c 2_{1}$ crystal structure about the central axis of the figure. The dashed lines show the interfaces between the six crystals.

We note, parenthetically, that it is straightforward to prove by similar methods that the only heterovalent ternary structures based on the zincblende lattice that are consistent with the octet rule are the polytypes identified and modeled by Wei et al. ${ }^{10}$ In that case, the structures are the $\mathrm{Cu}-\mathrm{Au}$ ordering (along [001]) and the chalcopyrite structure.

In the thermodynamic approach used in modeling polytypes, the differences in the free energies are the most important quantities. A major contribution to the free energy is the energy of formation, which is expressed for a crystal of $N$ pseudospin layers in terms of the layer interactions:

$$
E=E_{0}+\frac{1}{N} \sum_{n=1}^{M} \sum_{i=1}^{N-1} J_{n} s_{i} \cdot s_{i+n}
$$
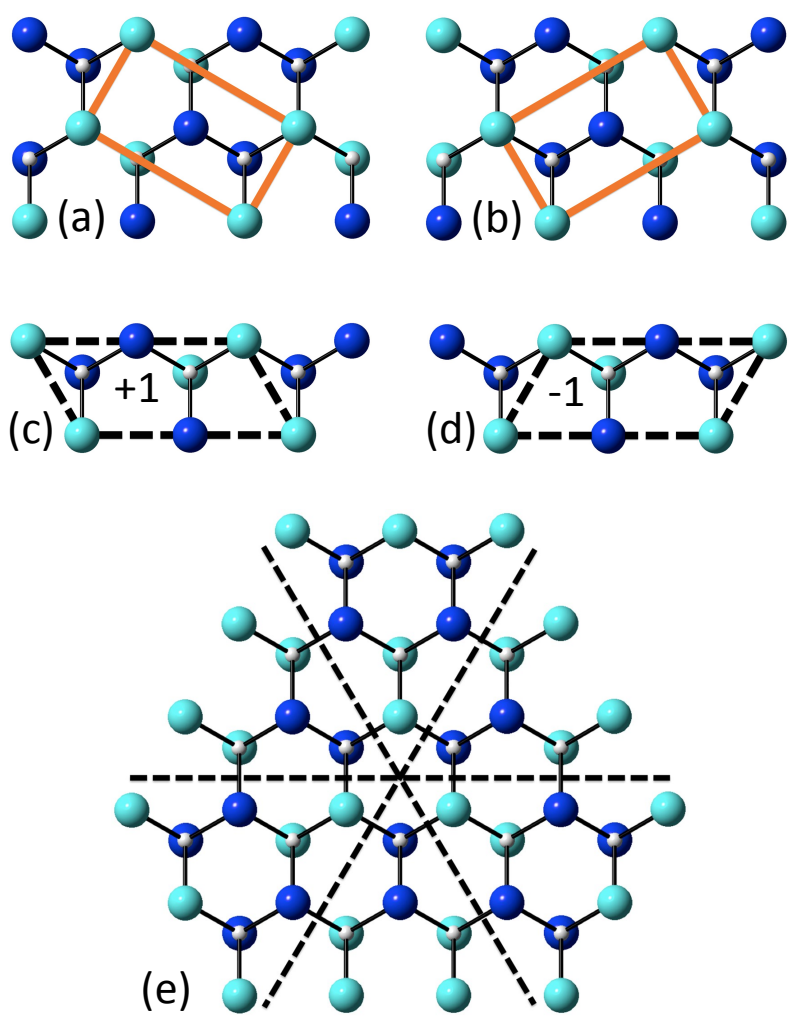

FIG. 2: (Color online) The $P m c 2_{1}$ crystal structure rotated, in (a) clockwise and (b) counterclockwise directions by $120^{\circ}$ with respect to Fig. 1, with the unit cell outlined; (c) defines the +1 and (d) the -1 pseudospin layer, with the 8 atom repeat units outlined in dashed black; (e) the octetrule-preserving twinned structure. The twin boundaries are shown by the dashed lines.

Here the $J_{n}$ are the energies of interaction between the $n$-th nearest-neighbor spin layers, with a range up to $M$, and $E_{0}$ is a convenient reference energy. The spins here are isospins pointing up or down. Non-trivial polytypes, i.e. long-range ordered patterns of spins, result from competition between different range interactions, as occurs for example in $\mathrm{SiC}$. In other cases, including the one considered here, the sign of $J_{1}$ determines which of the two alternative stackings has the lowest energy. No longrange polytypes have been observed for either $\mathrm{ZnSnN}_{2}$ or $\mathrm{ZnGeN}_{2}$, which suggests that for both of these cases the series can be restricted to nearest-neighbor interactions. We note that while this thermodynamic model can in principle predict the energetic ordering of different polytypes, in practice kinetics can play a decisive role in the formation of polytypes. 


\section{EXPERIMENTAL AND COMPUTATIONAL METHODS}

\section{A. First-principles calculations}

The calculations of the lattice parameters and total energy differences between different structures were carried out using a pseudopotential plane wave method as encoded in the abinit code. ${ }^{33-35}$ We used the Fritz-Haber pseudopotentials of Ref. 36. A high cut-off of 110 Hartree ensured convergence of the plane wave basis set. The $\mathbf{k}$ point mesh was chosen to be a shifted $4 \times 4 \times 4$ MonkhorstPack mesh for the $P n a 2_{1}$ structure. To have exactly equivalent energies, an $8 \times 4 \times 4$ mesh was used for the $P m c 2_{1}$ structure because that mesh has half the $a$-lattice constant and hence twice the size in that direction in reciprocal space. For the Pm31 structure, we relaxed the structure in the primitive four-atom cell but afterwards calculated the energy differences in the same cell as for the $P m c 2_{1}$ structure so that we could use exactly equivalent k-points. Calculations were performed both in the $\mathrm{LDA}^{37}$ and $\mathrm{GGA}^{30}$ approximations, which respectively underestimate and overestimate the lattice constants. This procedure allowed us to ascertain the effect of these errors on the energy differences. We found the errors to largely cancel out, which demonstrates that the LDA and GGA calculations provide consistent estimates of the energy differences.

The calculations of the energies of formation were performed using the $\mathrm{LDA}^{38-40}$ and using the full-potential linearized muffin-tin orbital (LMTO) band structure method. ${ }^{41}$ The energies of formation were defined with respect to the elements in their ground state phases at ambient conditions. For $\mathrm{N}$ this was with respect to the molecule $\mathrm{N}_{2}$, while for Ge and $\mathrm{Sn}$ it was with respect to the diamond structure, and for $\mathrm{Zn}$, with respect to the hcp phase. For Sn we used the $\alpha$-phase, which has the diamond structure and which is stable below $13.2^{\circ} \mathrm{C}$. Details of our calculations of the energies of formation can be found in Ref. 11. In particular, we point out that these calculations give acceptable accuracy for the cohesive energies and molecular binding energies of the individual elements, taking into account the usual overbinding of the LDA. Here we need only the differences in the energies of formation between the different structures considered. Any systematic errors due to LDA overbinding are expected to drop out of these differences. However, the contrast with previous work ${ }^{11,26}$ is that here we used structures that were fully relaxed with respect to $a$, $b$ and $c$ in addition to the internal atomic coordinates as obtained from the abinit method. This procedure gave us more accurate energies of formation, in particular for $\mathrm{ZnSnN}_{2}$, where the differences are small. We could of course also use the LMTO method to calculate the energy differences between the structures. However, convergence of the basis set to within the required precision of a few $\mathrm{meV}$ is harder to establish systematically. The differences agree with the abinit results on the order of a few tens of meV but we prefer to base our conclusions about the total energy differences on the abinit results, because these were deemed better converged with respect to the basis set.

The band structures were calculated using the QSGW approach, developed by van Schilfgaarde et al. ${ }^{25}$ and as applied to the current materials in Punya et al. ${ }^{26}$ This method gives gaps to a precision of about $0.1 \mathrm{eV}$ without any adjustable parameters for most tetrahedrally-bonded semiconductors. Because this method tends to underestimate the screening and hence overestimates the gaps typically by $20 \%$ for semiconductors, $80 \%$ of the self-energy was applied to the LDA calculation.

The calculations were done for the two crystal structures shown in Fig. 1 and for a four-atom unit cell with space group Pm31. This latter structure is composed of alternating planes of $\mathrm{A}$ and $\mathrm{B}$ cations stacked along the c-axis, and thus is an extreme case of the violation of local charge neutrality; all anions are bound to either three $\mathrm{A}$ atoms and one $\mathrm{B}$ atom, or vice versa.

\section{B. Calculation of the x-ray diffraction spectra}

We followed the formulation of Kopp et al. ${ }^{42}$ to calculate analytically the x-ray diffraction spectra for a $\mathrm{ZnSnN}_{2}$ crystal with one-dimensional disorder due to random stacking along the $\mathbf{b}$ axis, as a function of the total thickness of the crystal and as a function of the proportion of $P n a 2_{1}$ to $P m c 2_{1}$ stacking. This is a transfer matrix method that generates an analytical solution equivalent to a Monte Carlo generation of the averaged diffraction peak intensities for an ensemble of crystals. The solution is specific to the case of a random distribution of stacked layers described by a stationary Markov chain; that is, the probability of layer $i+1$ stacking upon layer $i$ is dependent only upon layer $i$. In this case, the probability is determined by specifying the relative proportions of the $P n a 2_{1}$ and $P m c 2_{1}$ phases.

The information required for the calculation includes the number of pseudospin layers $N$, the probability $N_{s} / N$ associated with each pseudospin type $s= \pm 1$, the structure factors for each of the pseudospin layers, and the conditional probabilities governing the likelihood of layer $j^{\prime}$ of pseudospin character $s_{j^{\prime}}$ occurring after layer $j$ with pseudospin character $s_{j}$. Because the two spin states are mirror-symmetric, they are equally probable, and thus $N_{+1} / N$ and $N_{-1} / N$ are both equal to $1 / 2$.

The structure factors for the two pseudospin layers were calculated using the atomic form factors for $\mathrm{Zn}, \mathrm{Sn}$, and $\mathrm{N}$ from the International Union of Crystallography database. The atomic positions were referenced to the orthorhombic lattice parameters $a=0.6749 \mathrm{~nm}, b=0.5845$ $\mathrm{nm}, c=0.55443 \mathrm{~nm}$ and the Wyckoff positions for the ideal wurtzite lattice.

The relative peak intensities were calculated using the standard Lorentz polarization factor $L_{p}(\theta)=(1+$ $\left.\cos ^{2} 2 \theta\right) /\left(\sin ^{2} \theta \cos \theta\right)$. While Kopp et al. ${ }^{42}$ avoided the 
Bragg vectors that resulted in singularities, we dealt with the singularities by replacing each reciprocal lattice vector $\mathbf{k}(\mathrm{h}, \mathrm{k}, \mathrm{l})$ with $\mathbf{k}^{\prime}=\mathbf{k}(h, k+\Delta, l)$, letting $\Delta=10^{-6}$.

\section{Experimental methods}

The polycrystalline $\mathrm{ZnSnN}_{2}$ material for the Raman and photoluminescence measurements was grown by a vapor-liquid-solid method described in Ref. 19. Briefly, the method involved the exposure to a nitrogen plasma of a $\mathrm{Zn}$-Sn melt held at $485{ }^{\circ} \mathrm{C}$. The grown material was a polycrystalline layer, approximately $300 \mathrm{~nm}$ thick, covering most of an area approximately $0.5 \mathrm{~cm}$ in diameter. The average crystallite diameter, estimated using the x-ray diffraction linewidths, was approximately $70 \mathrm{~nm}$. The measurement of the Raman spectrum was done in ambient conditions using a solid-state laser with photon energy at $532 \mathrm{~nm}$ focused to a 1-2 $\mu$ m diameter spot. Neither the incident nor the scattered radiation was polarization-resolved. The excitation source for the measurement of the photoluminescence spectrum was a $633 \mathrm{~nm}$ He-Ne laser with incident intensity $1.4 \mathrm{~kW} / \mathrm{cm}^{2}$. The measurement was done at $77^{\circ} \mathrm{K}$.

Polycrystalline $\mathrm{ZnGeN}_{2}$ samples were grown on $\mathrm{Ge}$ substrates exposed to $\mathrm{Zn}$ and $\mathrm{NH}_{3}$ at near-atmospheric pressure. The growth processes are described more fully in Ref. 29. X-ray diffraction measurements were done on the as-grown samples using a diffractometer in BraggBrentano configuration, with $\mathrm{Cu} \mathrm{K} \alpha(1,2)$ irradiation and a two-dimensional xenon detector with a step size of $0.02^{\circ}$. The instrumental broadening was measured to be $0.275 \pm 0.02^{\circ}$, using an alumina standard.

\section{RESULTS}

As mentioned in the introduction, the paradox that led us to abandon the model of a fully disordered cation sublattice was that $\mathrm{ZnSnN}_{2}$ was found to have a band gap close to that predicted for the perfectly ordered $P n a 2_{1}$ structure, while at the same time the material appeared to have a disordered wurtzite-like lattice structure. ${ }^{19}$ Because the $P n a 2_{1}$ and $P m c 2_{1}$ structures are superlattices of the wurtzite structure, distorted by deviations from the ideal wurtzite atomic positions, their x-ray diffraction spectra should be characterized by specific superlattice peaks absent in the disordered structure, and peak splittings arising from deviations in the ratios of the lattice parameters from the ideal wurtzite ratios. The strongest of the predicted superlattice peaks are the (101) peak for $P n a 2_{1}$ ordering and the (111) peak for $P m c 2_{1}$ ordering, as shown in Fig. 5(a). None of the superlattice peaks or expected peak splittings have been observed for $\mathrm{ZnSnN}_{2},{ }^{15,17,19}$ while superlattice peaks and the expected peak splittings for Pna2 $2_{1}$ ordering have been reported for $\mathrm{ZnGeN}_{2} \cdot{ }^{29}$ Because the calculated ratios of the lattice parameters are closer to the ratios for the ideal wurtzite lattice for $\mathrm{ZnSnN}_{2}$ than for $\mathrm{ZnGeN}_{2}$, as shown later in Table I, the splittings of the degeneracies in the diffraction peaks due to distortions from the idealized wurtzite structure are expected to be larger for $\mathrm{ZnGeN}_{2}$ than for $\mathrm{ZnSnN}_{2}$. On the other hand, the superlattice peak intensities depend on the differences in the atomic form factors between the group-IV and group-II cations. These are larger for Sn relative to $\mathrm{Zn}$ than for Ge relative to $\mathrm{Zn}$, and consequently the predicted superlattice peak intensities for ordered $\mathrm{ZnSnN}_{2}$ are roughly an order of magnitude larger than for ordered $\mathrm{ZnGeN}_{2}$.

In the first subsection we present additional evidence from Raman spectroscopy that the lattice of $\mathrm{ZnSnN}_{2}$ is indeed disordered, and that the band gap is close to 1.7 $\mathrm{eV}$. In the second subsection we present the computational results for the lattice parameters, the energies of formation, and the band gaps of the different ordered structures. Comparison of the band gaps and energies of formation shows that violations of the octet rule cost substantial energy and lower the band gaps. Finally, we apply the pseudospin model to the calculation of the $\mathrm{x}$ ray diffraction spectra of $\mathrm{ZnSnN}_{2}$ for different numbers of layers and proportions of the $P n a 2_{1}$ and $P m c 2_{1}$ phases, and resolve the apparent paradox involving the band gap energy and x-ray diffraction spectra of $\mathrm{ZnSnN}_{2}$.

\section{A. Photoluminescence and Raman spectroscopy}

Previously reported photoluminescence excitation (PLE) spectroscopy of $\mathrm{ZnSnN}_{2}$ done at room temperature yielded an estimated band gap of $1.7 \pm 0.1 \mathrm{eV}$ that was measured using a broad defect luminescence peak centered at $1.5 \mathrm{eV}$. At room temperature there was no near-band-edge luminescence peak present. ${ }^{19}$ At $77^{\circ} \mathrm{K}$ the defect peak at $1.5 \mathrm{eV}$ is still evident (Fig. 3(a)), but the photoluminescence spectrum is dominated by the near-band-edge peak centered at approximately 1.72 $\mathrm{eV}$. The relative increase in the near-band-edge luminescence at the lower temperature, a commonly-observed phenomenon, results from the slowing of the transfer of photoexcited carriers to the defect states responsible for radiative recombination. The band gap inferred from the $77^{\circ} \mathrm{K}$ photoluminescence spectrum is consistent with the octet-rule-preserving model of disorder and is inconsistent with a model for the disorder that violates the octet rule. Fig. 3(b) shows the dependence of the defect and near-band-edge peak intensities as a function of excitation intensity, with the defect peak height normalized to that of the near-band-edge peak at the lower range of excitation intensity, in order to best compare the dependence of the two peak intensities on excitation intensity. At lower excitation intensity the dependence is linear, while at higher intensity the dependence becomes superlinear, an indication that defect recombination channels are becoming saturated at this still-low range of excitation intensity. Deconvolution of the two peaks proved unnecessary to obtain the defect peak intensity accurately, 

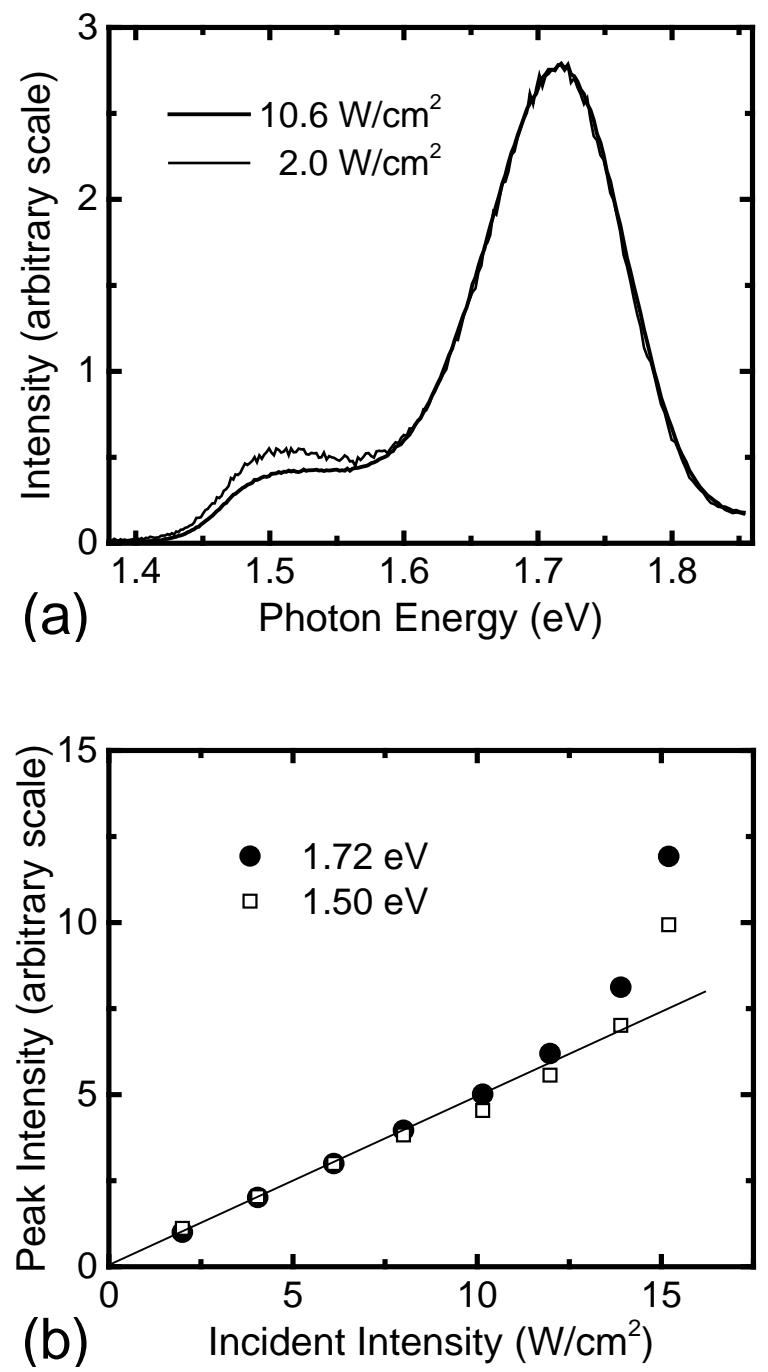

FIG. 3: (a) Photoluminescence spectrum of $\mathrm{ZnSnN}_{2}$ at 77 ${ }^{\circ} \mathrm{K}$. The peak at $1.72 \mathrm{eV}$ is near-band-edge recombination. The peak at $1.5 \mathrm{eV}$ is defect recombination, as was identified in previous work. ${ }^{19}$ (b) Photoluminescence peak intensities versus incident intensity. The defect peak intensity at $1.5 \mathrm{eV}$ has been scaled to the peak intensity of the $1.72 \mathrm{eV}$ nearband-edge peak intensity at low excitation intensities.

as the near-band-edge peak intensity at $1.5 \mathrm{eV}$ was negligible.

We turn now to the results of calculations and measurement of the Raman spectrum of $\mathrm{ZnSnN}_{2}$. The calculated Raman spectrum in Fig.4(a) was obtained by adding the calculated intensities of all modes $\left(a_{1}, b_{1}, b_{2}\right.$ and $\left.a_{2}\right)$, with equal weights, taken from Ref. 43 . This spectrum is dominated by a peak at approximately $590 \mathrm{~cm}^{-1}$, identified with a wurtzite- $E_{2}$-high-like vibrational pattern. The measured Raman spectrum of Fig. 4(b) shows none of the predicted Raman peaks, not even the dominant one.

We emphasize that this result is quite different from

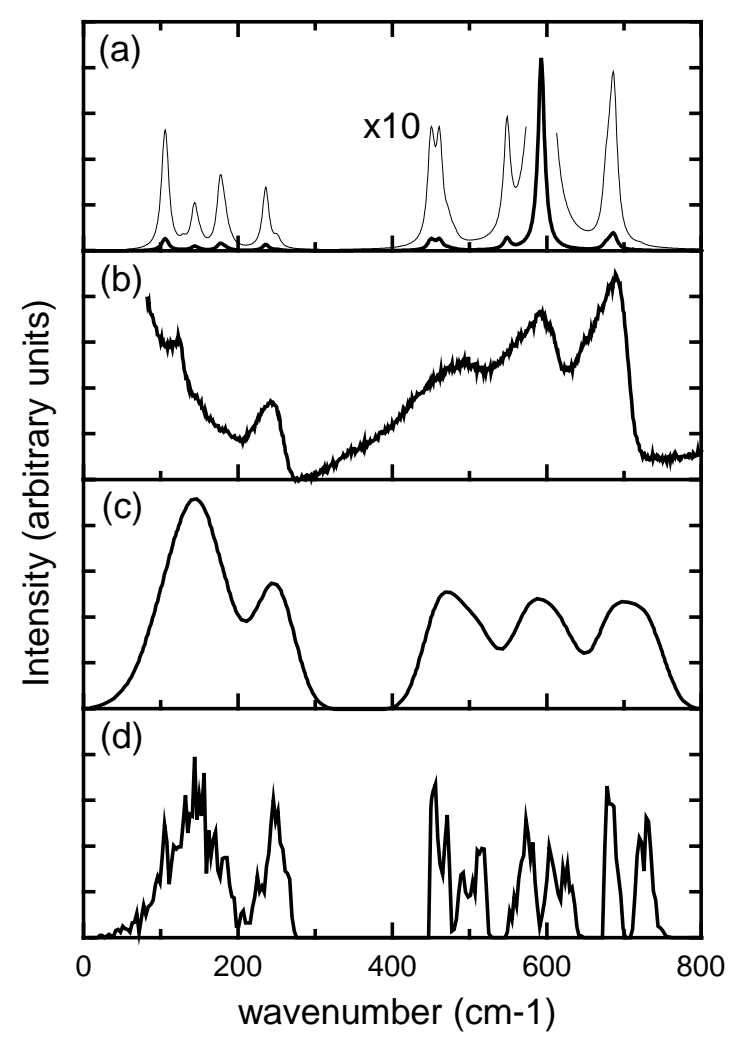

FIG. 4: Raman spectra and calculated phonon density of states of $\mathrm{ZnSnN}_{2}$. Calculated (a) and measured (b) Raman spectra. Calculated density of states, (c) Gaussianbroadened, and (d) high-resolution.

the Raman spectra of polycrystalline $\mathrm{ZnGeN}_{2}$, for which all of the predicted Raman peaks were observed. Instead, the $\mathrm{ZnSnN}_{2}$ Raman spectrum closely resembles the phonon density-of-states spectrum, shown Gaussianbroadened in Fig. 4(c) and with high energy resolution in Fig. 4(d), although the peak shapes are somewhat different. The experimental spectrum is strikingly phononglass-like, showing complete breakdown of the $\mathbf{k}$-vector selection rule. This result is consistent with the $\mathrm{x}$-ray diffraction results showing a highly disordered lattice. However, the Raman spectrum does not give information on the type of disorder, and in particular does not, in the absence of quantitative modeling, distinguish between our model for disorder due to random stacking of pseudospin layers and one for which the cations are fully disordered on the wurtzite lattice.

\section{B. Results of first-principles calculations}

In Table I we compare our calculated lattice constants, for different computational methods and different structures, with measured results. The calculated lattice constants were obtained using the abinit code with full re- 
laxation of the structure. As an average measure of the uncertainty we use $((\delta a / a)(\delta b / b)(\delta c / c))^{1 / 3}=(\delta V / V)^{1 / 3}$, with $\delta a$ the deviation from experiment for the $a$ lattice constant, etc. We see that the LDA calculations underestimate the lattice constants of $\mathrm{ZnGeN}_{2}$ by $1.6 \%$ and those of $\mathrm{ZnSnN}_{2}$ by $2.4 \%$. On the other hand, the GGAcalculations overestimate the lattice constants by $1.3 \%$ for $\mathrm{ZnGeN}_{2}$ and $1.1 \%$ for $\mathrm{ZnSnN}_{2}$. These are typical errors for the LDA and GGA methods. Within each of these methods the predicted lattice constants for the $P m c 2_{1}$ and $P n a 2_{1}$ structures are the same, on average, to within $0.1 \%$, for both materials. While there are some larger differences in the individual lattice constants, the volumes agree to about $0.3 \%$. Similar results are obtained for the Pm31 structure, which has a slightly larger unit cell volume. For $\mathrm{ZnGeN}_{2}$, both $b / a$ and $c / a$ are larger by about $6 \%$ and $3 \%$, respectively, relative to the $P n a 2_{1}$ structure, but the $a$ lattice parameter is smaller. The lattice parameters for the two $\mathrm{ZnSnN}_{2}$ structures are closer than for $\mathrm{ZnGeN}_{2}$, as expected, since the $\mathrm{Zn}-\mathrm{N}$ and $\mathrm{Sn}-\mathrm{N}$ bonds are closer in length than are the $\mathrm{Zn}-\mathrm{N}$ and Zn-Ge bonds.

The energies in Table II were calculated using the relaxed lattice constants for each phase. We see from Table II that the difference in the energy of formation between $P m c 2_{1}$ and $P n a 2_{1}$ is extremely small in $\mathrm{ZnSnN}_{2}$, amounting to only $16 \mathrm{meV}$ per formula unit in the LDA calculations. The LDA and GGA calculations agree on this small difference to within a few $\mathrm{meV}$ precision. In contrast, the difference between the energies of formation of the Pm31 and the Pna2 $2_{1}$ structures is over 0.5 $\mathrm{eV}$, taking the average of the LDA and GGA calculations. This result confirms that the two structures that obey the octet rule are negligibly different in their energies of formation while the structure with only octet-ruleviolating tetrahedra has signficantly larger total energy. For $\mathrm{ZnGeN}_{2}$, although the $P m c 2_{1}$ and $P n a 2_{1}$ structures differ in total energy by about an order of magnitude more than for $\mathrm{ZnSnN}_{2}$ (about $0.12 \mathrm{eV} /$ formula unit), the introduction of octet-rule-violating tetrahedra costs substantially more energy (over $2 \mathrm{eV}$ ).

Before leaving the topic of the differences in energies of formation, we note that our calculated energy of formation for $\mathrm{ZnSnN}_{2}$ in the $P n a 2_{1}$ structure, with respect to the elements, is $-0.734 \mathrm{eV} /$ formula unit. This value differs from the value quoted in Ref. 11 and is now in better agreement with, but still more negative than, that of Chen et al. ${ }^{20}$ The change is mainly due to the more accurate structural relaxation in the present work.

Turning now to the band gaps, we see from the last column in Table II that the band gaps in the Pna2 ${ }_{1}$ and $P m c 2_{1}$ structures are close to each other, and those of the Pm31 structures are much smaller than these. In fact, for $\mathrm{ZnSnN}_{2}$ we find a metallic band structure. The full band structures of $\mathrm{ZnSnN}_{2}$ and $\mathrm{ZnGeN}_{2}$ in the three structures are shown in Figs. 5 and 6 . The symmetry points used in these plots correspond to $\Gamma=(0,0,0), X=(\pi / a, 0,0)$, $Y=(0, \pi / b, 0), Z=(0,0, \pi / c), U=(\pi / a, 0, \pi / c)$,
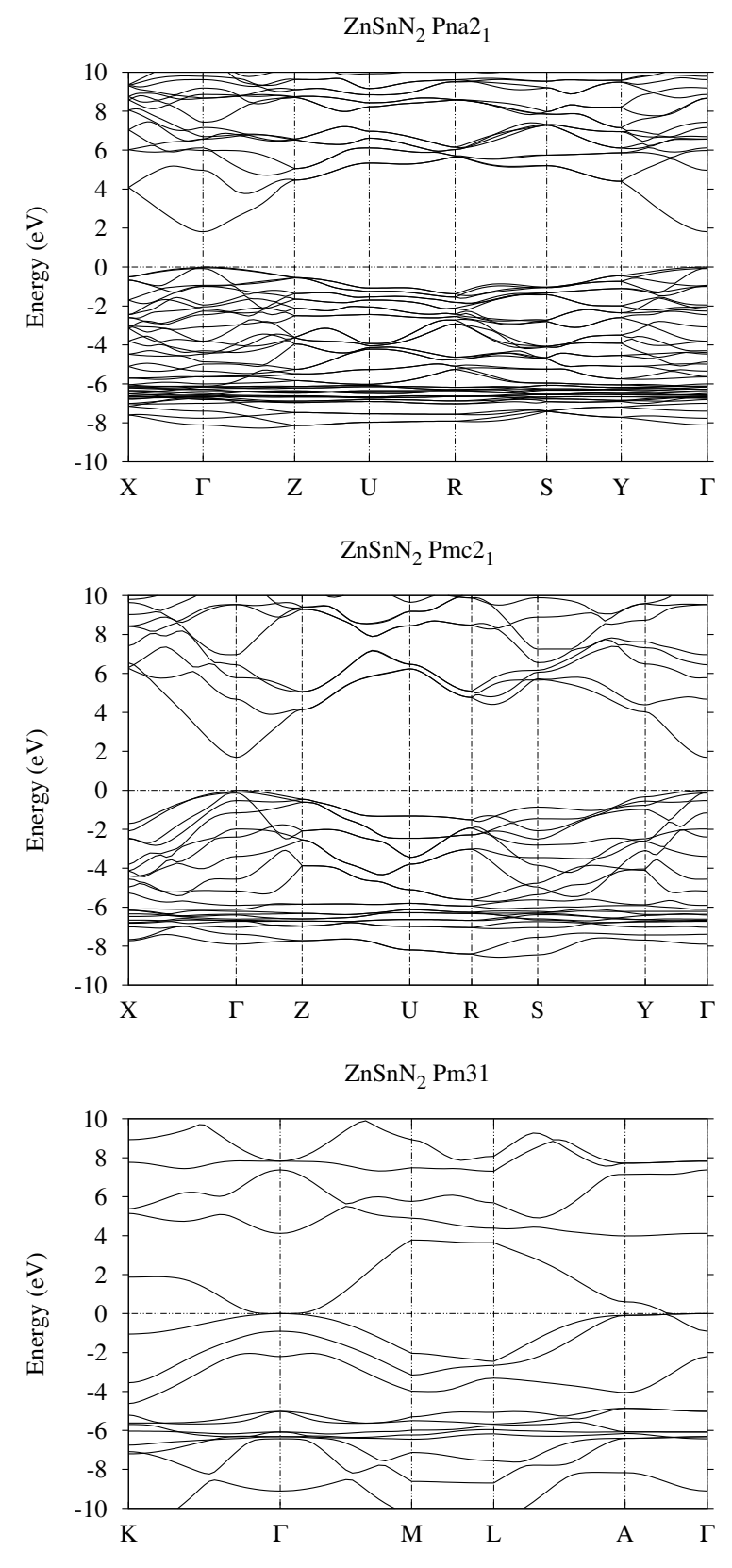

FIG. 5: Band structures of $\mathrm{ZnSnN}_{2}$ in, from top to bottom, the $P n a 2_{1}, P m c 2_{1}$ and Pm31 structures.

$R=(\pi / a, \pi / b, \pi / c)$ and $S=(\pi / a, \pi /, 0)$ for the orthorhombic cell and the standard ones for the trigonal structure, similar to those in wurtzite. We can see that $\mathrm{ZnSnN}_{2}$ in the Pm31 structure has an inverted band structure. Because of the two-fold degenerate valence band maximum the inversion will open a small gap of order meV when spin-orbit coupling is included. In that sense we reported the gap as zero in the Table II. For $\mathrm{ZnGeN}_{2}$ the difference in the band gaps of the $P m c 2_{1}$ and $P n a 2_{1}$ structures is $-0.65 \mathrm{eV}$ while for $\mathrm{ZnSnN}_{2}$ it is only $-0.12 \mathrm{eV}$. The reason for the larger difference in band gaps for $\mathrm{ZnGeN}_{2}$ probably lies in the larger difference in 
TABLE I: Lattice constants in $\AA$ and unit cell volumes in $\AA^{3}$ for $\mathrm{ZnGeN}_{2}$ and $\mathrm{ZnSnN}_{2}$ for the different structures and computational methods, compared with experiment. Note that the ideal wurtzite $b / a_{w}$ and $c / a_{w}$ ratios are $b / a=\sqrt{3} \sim 1.732$ and $c / a=\sqrt{8 / 3} \sim 1.633$.

\begin{tabular}{|c|c|c|c|c|c|c|c|c|}
\hline compound & structure & method & $a$ & $b$ & $c$ & $b / a_{w}$ & $c / a_{w}$ & $V$ \\
\hline \multirow{7}{*}{$\mathrm{ZnGeN}_{2}$} & $P n a 2_{1}$ & Expt. $^{a}$ & 6.44 & 5.45 & 5.19 & 1.693 & 1.612 & 182.16 \\
\hline & & LDA & 6.327 & 5.358 & 5.115 & 1.694 & 1.617 & 173.40 \\
\hline & & GGA & 6.521 & 5.522 & 5.264 & 1.694 & 1.615 & 189.55 \\
\hline & $P m c 2_{1}$ & LDA & $6.102^{b}$ & 5.532 & 5.127 & 1.813 & 1.680 & 173.07 \\
\hline & & GGA & 6.294 & 5.699 & 5.275 & 1.801 & 1.676 & 189.21 \\
\hline & Pm31 & LDA & $6.238^{c}$ & 5.402 & 5.238 & 1.732 & 1.679 & 176.48 \\
\hline & & GGA & 6.447 & 5.581 & 5.388 & 1.731 & 1.671 & 193.89 \\
\hline \multirow[t]{7}{*}{$\mathrm{ZnSnN}_{2}$} & $P n a 2_{1}$ & Expt. $^{d}$ & 6.753 & 5.842 & 5.462 & 1.730 & 1.618 & 215.48 \\
\hline & & LDA & 6.573 & 5.698 & 5.342 & 1.733 & 1.625 & 200.07 \\
\hline & & GGA & 6.812 & 5.905 & 5.534 & 1.734 & 1.625 & 222.60 \\
\hline & $P m c 2_{1}$ & LDA & 6.562 & 5.689 & 5.344 & 1.734 & 1.629 & 199.50 \\
\hline & & GGA & 6.804 & 5.896 & 5.536 & 1.733 & 1.627 & 222.08 \\
\hline & $P m 31$ & LDA & 6.604 & 5.718 & 5.340 & 1.732 & 1.617 & 201.62 \\
\hline & & GGA & 6.848 & 5.929 & 5.544 & 1.732 & 1.619 & 225.07 \\
\hline
\end{tabular}

${ }^{a}$ From Ref. 27

${ }^{b}$ Note that for the $P m c 2_{1}$ structure, this parameter is twice the $P m c 2_{1}$ lattice constant $a_{o^{\prime}}$.

${ }^{c}$ Note that we give the lattice constants corresponding to those for the Pna2 1 structure.

${ }^{d}$ From Ref. 19

TABLE II: The energies of formation per formula unit (fu) relative to the energies of formation of the $P n a 2_{1}$ structures, and the band gaps $E_{g}$. The labels LDA and GGA in the column method only refer to the total energy differences, the gaps were obtained in the QSGW method using $0.8 \Delta \Sigma$ at the relaxed lattice constants for each phase in the LDA.

\begin{tabular}{|c|c|c|c|c|}
\hline compound & structure & method & $\Delta E_{t o t}(\mathrm{eV} / \mathrm{fu})$ & $E_{g}(\mathrm{eV})$ \\
\hline \multirow{5}{*}{$\mathrm{ZnGeN}_{2}$} & $P n a 2_{1}$ & & 0 & 3.65 \\
\hline & $P m c 2_{1}$ & LDA & 0.120 & 3.00 \\
\hline & & GGA & 0.111 & \\
\hline & $\operatorname{Pm} 31$ & LDA & 0.945 & 1.21 \\
\hline & & GGA & 0.815 & \\
\hline \multirow[t]{5}{*}{$\mathrm{ZnSnN}_{2}$} & $P n a 2_{1}$ & & 0 & 1.81 \\
\hline & $P m c 2_{1}$ & LDA & 0.016 & 1.69 \\
\hline & & GGA & 0.011 & \\
\hline & $\operatorname{Pm} 31$ & LDA & 0.564 & 0 \\
\hline & & GGA & 0.464 & \\
\hline
\end{tabular}

the bond lengths of the Zn-N and Ge-N bonds, which are affected by the slightly different structural relaxations in both crystal structures. We did not include here the zeropoint motion corrections, which are of order $0.1 \mathrm{eV}$, or the exciton binding energy corrections, which are of order $20 \mathrm{meV}$, as was done in Punya et al. ${ }^{26}$ These corrections were calculated using the LMTO QSGW approach but using the LDA structure obtained in abinit and should be roughly the same for the $P m c 2_{1}$ and $P n a 2_{1}$ structures so should not affect the differences in those gaps. For semiconductors, the pure QSGW calculation underestimates the screening of the electron-electron interaction by about $20 \%$, so we used $0.8 \Delta \Sigma$ to compensate for this underestimate, where $\Delta \Sigma$ is the difference between the $G W$ self-energy and the LDA exchange correlation potential. We estimate the total uncertainty in the calculations of the band gaps to be of order $0.1 \mathrm{eV}$, including the uncertainty due to the underestimate of the lattice constants.

We now interpret the reduction in the band gap of Pm31 in terms of the presence of octet-rule-violating local environments. This reduction in band gap is even greater than the approximately $1 \mathrm{eV}$ difference reported by Feldberg et al. ${ }^{15}$ Similar trends were reported in the comparison of the calculated band gaps and total energies of the ordered and disordered ternary zincblendebased $\mathrm{ZnSnP}_{2}$ system, with larger band gaps and smaller energies of formation for the two ordered, octet-rulepreserving $\mathrm{CuAu}$ and chalcopyrite structures, compared to an ordered octet-rule-violating structure, and to a structure with $\mathrm{Sn}$ and $\mathrm{Zn}$ atoms placed randomly on the cation sublattice. ${ }^{44}$ For the $\mathrm{ZnSnN}_{2}$ system, the defect calculations by Chen et $a .^{20}$ also provide some insight, although less directly, into the reductions in band gaps that can be expected from violations of the octet rule. They report $\mathrm{Sn}_{\mathrm{Zn}}$ antisite defects to have a $+2 / 0$ transition level at about $0.2 \mathrm{eV}$ below the gap, while $\mathrm{Zn}_{\mathrm{Sn}}$ antisites show a $0 / 2-$ transition level at about $0.6 \mathrm{eV}$ above the valence band maximum. The defects violate the octet rule in the sense that they lead locally to tetrahedra with $1 \mathrm{Zn}$ and $3 \mathrm{Sn}$ or vice versa. Although we cannot directly identify Kohn-Sham levels of defects with transition levels, the two should be rather closely related. At a sufficiently large concentration the antisite defect levels would broaden into bands and may thus be expected to reduce 

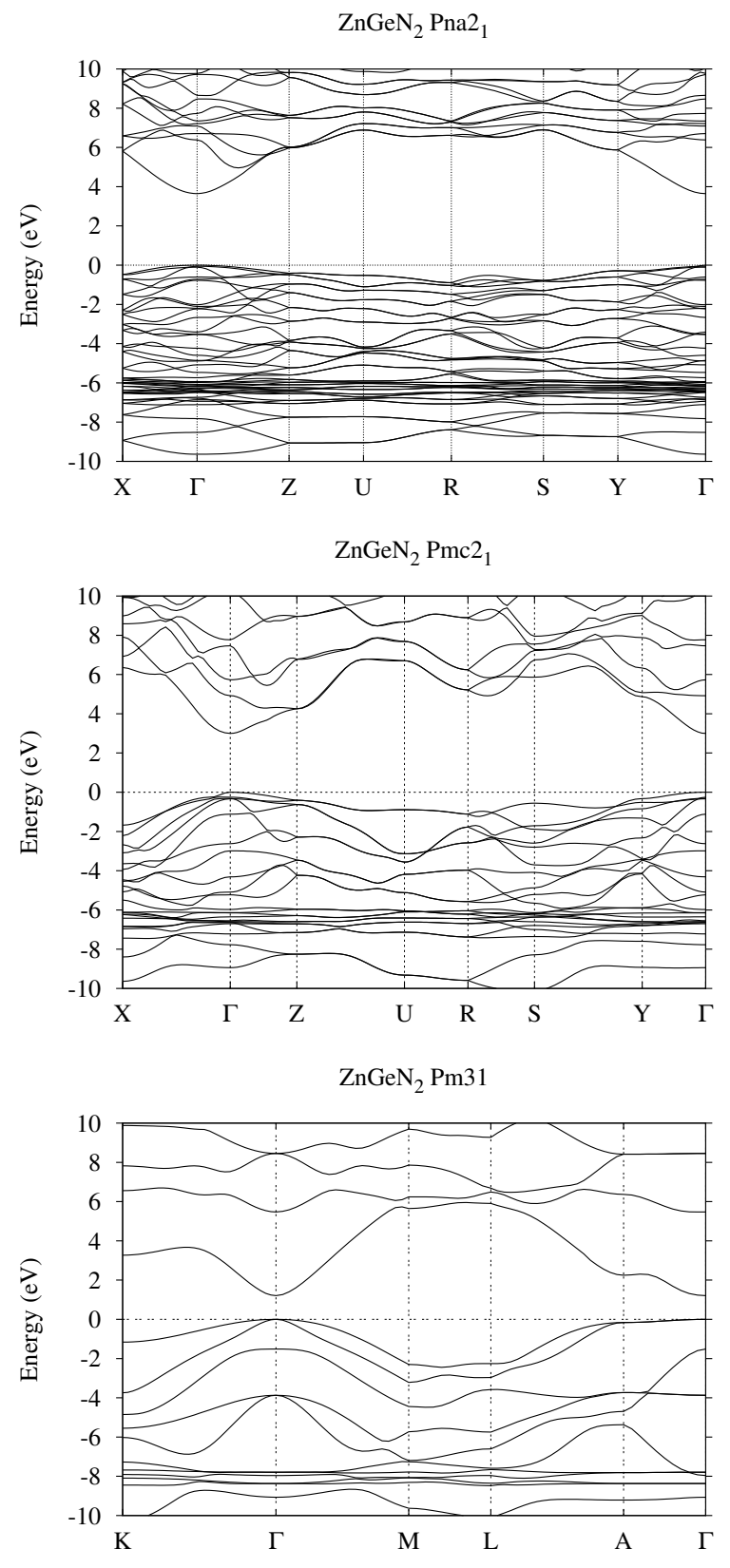

FIG. 6: Band structures of $\mathrm{ZnGeN}_{2}$ in, from top to bottom, the $P n a 2_{1}, P m c 2_{1}$ and $P m 31$ structures.

the band gap. Thus, Chen et al.'s results ${ }^{20}$ suggest that octet-rule-violating antisite defects could reduce the gap by about $0.8 \mathrm{eV}$.

The Boltzmann factors for $P m c 2_{1^{-}}$and Pna2 $2_{1}$-type stackings are $e^{-\Delta E_{f} / k T} /\left(1+e^{-\Delta E_{f} / k T}\right)$ and $1 /(1+$ $\left.e^{-\Delta E_{f} / k T}\right)$, respectively. These factors do not take properly into account the statistics of the one-dimensional stacking, the energy costs and entropy of the octet-ruleviolating defects accompanying disruptions in the ordering within a layer, the thermodynamics at the growth interface, which can be quite different from in the bulk, and, perhaps most importantly, the kinetics of the growth and ordering processes. However, they may give a very rough indication of how likely the two phases are to mix. For a growth temperature of about $750{ }^{\circ} \mathrm{K}$ for $\mathrm{ZnSnN}_{2}$, the Boltzmann factors give proportions of the $P m c 2_{1}$ - to Pna2 $2_{1}$-type orderings of $55 \%$ and $45 \%$. The closeness of the lattice constants indicates that strain effects on the gap will be negligible. The band offsets between the two phases are also expected to be quite small because the valence band maxima in both structures consist of $\mathrm{N}-2 p$ like states. Thus, for $\mathrm{ZnSnN}_{2}$ we should expect random stackings of close to equal proportions of the two phases, and a band gap approximately independent of the ordering as long as octet-rule-violating defects are avoided. The $0.12 \mathrm{eV}$ difference in the two band gaps, plus any small effects of strain and band offsets, will result in the generation of a random short-period superlattice that may be responsible for the roughly $120 \mathrm{meV}$ linewidth of the near-band-edge luminescence peak observed in Fig. 3.

For $\mathrm{ZnGeN}_{2}$, the difference in the energies of formation for the $P n a 2_{1}$ and $P m c 2_{1}$ structures $(\sim 0.12 \mathrm{eV})$ is over an order of magnitude larger than for $\mathrm{ZnSnN}_{2}$ but the growth temperature $\left(\sim 1000{ }^{\circ} \mathrm{K}\right)$ is also larger. The Boltzmann factors give about $80 \%$ Pna2 $2_{1}$ and $20 \%$ $P m c 2_{1}$. Thus, for $\mathrm{ZnGeN}_{2}$ there should be a significantly lower tendency for mixing of the two phases to occur. However, it should be emphasized that these Boltzmann factors should not be used to predict the proportions of the two phases, for the reasons noted above.

\section{Calculated x-ray diffraction spectra}

With values for the energies of formation obtained by calculation, we can now calculate the layer interaction parameter $J_{1}$ defined in Eq.(1); we have $E_{f}\left(P n a 2_{1}\right)-$ $E_{f}\left(P m c 2_{1}\right)=-2 J_{1}$. For $\mathrm{ZnSnN}_{2}$ we obtain $J_{1}=$ $6.5 \pm 1.5 \mathrm{meV}$, and for $\mathrm{ZnGeN}_{2}, J_{1}=60 \pm 5 \mathrm{meV}$. As mentioned previously, we can neglect longer range interactions in the absence of evidence of polytypes of these materials. For $\mathrm{ZnSnN}_{2}$, the small value of $J_{1}$, compared to $\mathrm{kT}$ at the growth temperature, is consistent with roughly equal probabilities for the two types of stacking.

We now show that for $\mathrm{ZnSnN}_{2}$ the measured, wurtzitelike x-ray diffraction spectra are consistent with our ordering model. Figure 7 shows the calculated x-ray diffraction spectra for $\mathrm{ZnSnN}_{2}$ for four cases; for the perfectly ordered $P n a 2_{1}$ and $P m c 2_{1}$ phases, for a disordered wurtzite phase for which the atomic form factor for the cations is the average of those of $\mathrm{Zn}$ and $\mathrm{Sn}$, and for a crystal composed of 240 pseudospin layers made up of equal proportions, but random distributions, of +1 and -1 pseudospins. For ease of comparison we use the ideal wurtzite atomic positions, the lattice parameter $a=0.671 \mathrm{~nm}$, and the ideal wurtzite ratios $b / a=\sqrt{3}$ and $c / a=\sqrt{8 / 3}$. The strongest superstructure peaks unique to the pure crystals, which are the (111) peak for the $P m c 2_{1}$ structure and the (101) peak for the $P n a 2_{1}$ 


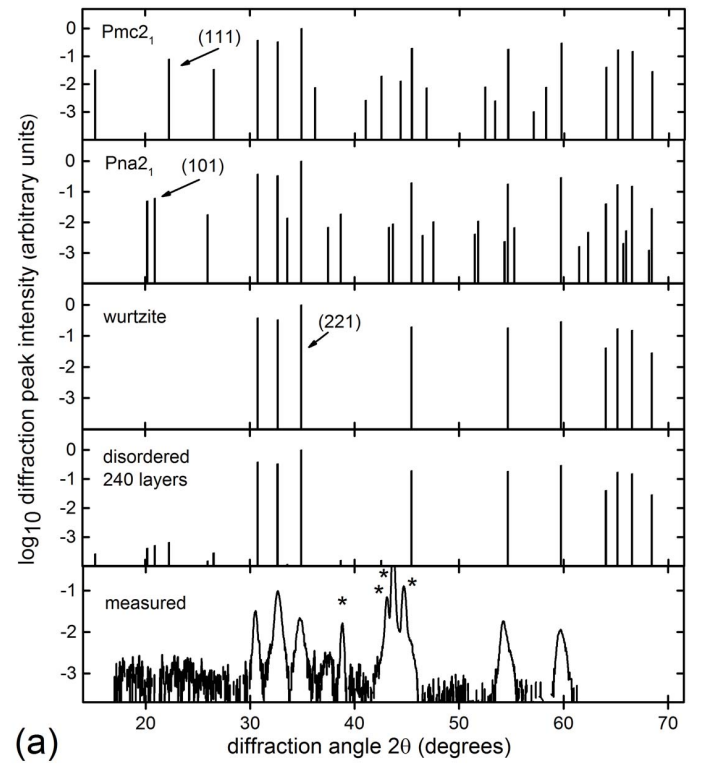

FIG. 7: $\mathrm{X}$ ray diffraction spectra for $\mathrm{ZnSnN}_{2}$. From top to bottom: the calculated powder x-ray diffraction spectra of $\mathrm{ZnSnN}_{2}$ for the $P n a 2_{1}, P m c 2_{1}$, and wurtzite crystal structures, a crystal composed of 240 pseudospin layers with a 50:50 mixture of the $P n a 2_{1}$ and $P m c 2_{1}$ structures, and the measured spectrum, from Ref. 19. The starred peaks in the measured spectrum are associated with the $\mathrm{Zn}$-Sn melt upon which the $\mathrm{ZnSnN}_{2}$ was grown.

structure, are labeled. Note that these are reduced in intensity by two orders of magnitude for the 240-layer disordered crystal, compared to the perfectly ordered structures. Superstructure peaks are absent in the measured spectrum, from Ref. 19, and in the other reports of x-ray diffraction measurements of $\mathrm{ZnSnN}_{2} \cdot{ }^{15,17}$

Figure 8(a) shows the dependence of the intensities of the superstructure peaks on the number of layers, relative to the $P n a 2_{1}$ (221) peak, which is the strongest peak common to the $P n a 2_{1}, P m c 2_{1}$ and wurtzite crystal structures. This result illustrates that the measurement of the diffraction spectrum as a function of the number of layers should be a powerful method to probe the disorder. The calculations also show that the x-ray diffraction spectra in references 17 and 19 have signal-to-noise ratios far too low to detect the superstructure peak intensities predicted for this model. In the first case, the depth probed is the typical $\mathrm{Cu} \mathrm{K} \alpha$ coherence length. In the second case, the coherence length is limited by the 70 $\mathrm{nm}$ grain size of the polycrystalline material.

Figure 8 (b) shows the result of a calculation of the highest-intensity superstructure peaks, for both a $240-$
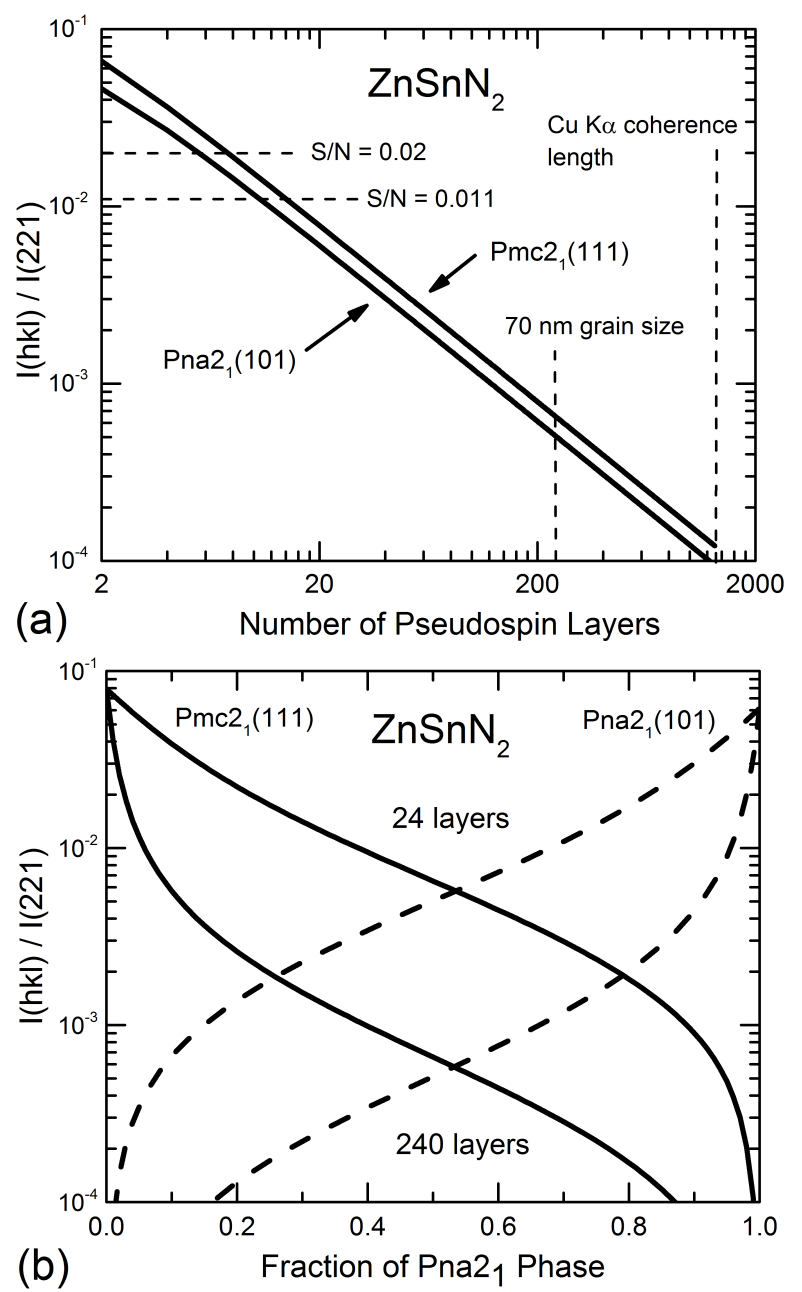

FIG. 8: Normalized $\mathrm{x}$ ray diffraction peak intensities for $\mathrm{ZnSnN}_{2}$. (a) The ratio of the $P m c 2_{1}$ (111) peak to the (221) peak for a 50:50 mixture of the $P n a 2_{1}$ and $P m c 2_{1}$ phases, as a function of the number of layers. The intensity of the (221) peak is independent of the ratio of the two phases. The two vertical dashed lines mark the number of layers that correspond to the average grain size reported in Ref.19, and the typical coherence length of a standard $\mathrm{Cu} \mathrm{K} \alpha$ x-ray source. The two horizontal dashed lines at 0.02 and 0.011 mark the signal-to-noise ratios for the $\mathrm{ZnSnN}_{2}$ x-ray diffraction spectra reported in references 17 and 19 , respectively. (b) The ratios of the $P n a 2_{1}$ (111) and $P m c 2_{1}$ (101) x-ray diffraction peaks to the (221) peak versus the fractional mixtures of the two phases, calculated for sampling depths of 24 and 240 pseudospin layers.

layer crystal and for a 24-layer crystal, as the proportions of the two pure phases are varied. It is clear from this figure that a study of the diffraction peak intensities as a function of growth conditions could provide important information on growth kinetics and thermodynamics. The figure also illustrates that the relative intensity of the superlattice diffraction peak to the Pna2 $2_{1}$ (221) peak drops very quickly below the signal-to-noise ratio unless the stacking is nearly perfect $P n a 2_{1}$ or $P m c 2_{1}$, or 
unless only a few layers in the crystal are sampled.

Unlike in the case of $\mathrm{ZnSnN}_{2}$, for which all measurements to date show a fully disordered lattice, it has been shown that for $\mathrm{ZnGeN}_{2}$ the extent of the lattice disorder may be tuned by growth conditions. Figure 9 shows two measured powder diffraction spectra for polycrystalline $\mathrm{ZnGeN}_{2}$ grown at different temperatures but under otherwise identical conditions. ${ }^{29}$ The spectrum for the material grown at $840^{\circ} \mathrm{C}$ is well represented by the calculated spectrum for the Pna2 1 phase, while the spectrum for the material grown at $750^{\circ} \mathrm{C}$ is consistent with the calculation for the fully disordered material. The effect of the distortion of the $P n a 2_{1}$ lattice from the ideal wurtzite is evident in the comparison of the simulated $\mathrm{ZnGeN}_{2}$ Pna2 $2_{1}$ and wurtzite spectra; the distortion results in the breaking of degeneracies for many of the diffraction peaks. That the x-ray diffraction spectra show disordered $\mathrm{ZnGeN}_{2}$ at the lower temperature and ordering in the $P n a 2_{1}$ phase at the higher temperature indicates that at $750^{\circ} \mathrm{C}$ the cations, more strongly bound than in $\mathrm{ZnSnN}_{2}$, do not have sufficient mobility to rearrange themselves on the cation sublattice. This result is not a signature of an order-disorder transition, for which the disordered phase appears at the higher, not lower, temperature, but is most likely a result of the limiting kinetics.

Finally, a very rough measure of the expected temperature for the transition from the lowest-energy ordered structure to a random stacking of the two structures can be given by the difference in the energies of formation of the Pna2 $2_{1}$ and $P m c 2_{1}$ structures, divided by Boltzmann's constant. For $\mathrm{ZnGeN}_{2}$ this estimate is of order $1400{ }^{\circ} \mathrm{K}$, and for $\mathrm{ZnSnN}_{2}, 156{ }^{\circ} \mathrm{K}$. Thus, for $\mathrm{ZnGeN}_{2}$ the growth temperature is well below this transition temperature, while for $\mathrm{ZnSnN}_{2}$ the growth temperature is well above the transition temperature, consistent with the qualitative differences in the amount of stacking disorder observed in the two cases.

\section{SUMMARY AND OUTLOOK}

We have shown that all octet-rule-preserving arrangements of the atoms in heterovalent ternary materials related to the binary wurtzite structure are described by the one-dimensional stacking of two pseudospin layers, with the exception of a unique, highly symmetric, multiply twinned structure.

For $\mathrm{ZnSnN}_{2}$, we have calculated the x-ray diffraction spectra of random stackings of the pseudospin layers as functions of the numbers of layers and the proportions of the pure materials. The results illustrate that x-ray diffraction methods can be powerful tools for probing this type of one-dimensional stacking disorder. Firstprinciples calculations of the energies of formation and band gaps of the constituent pure crystal structures for $\mathrm{ZnSnN}_{2}$ show that the charge-neutral $P n a 2_{1}$ and $P m c 2_{1}$ phases have very similar band gaps, lattice parameters

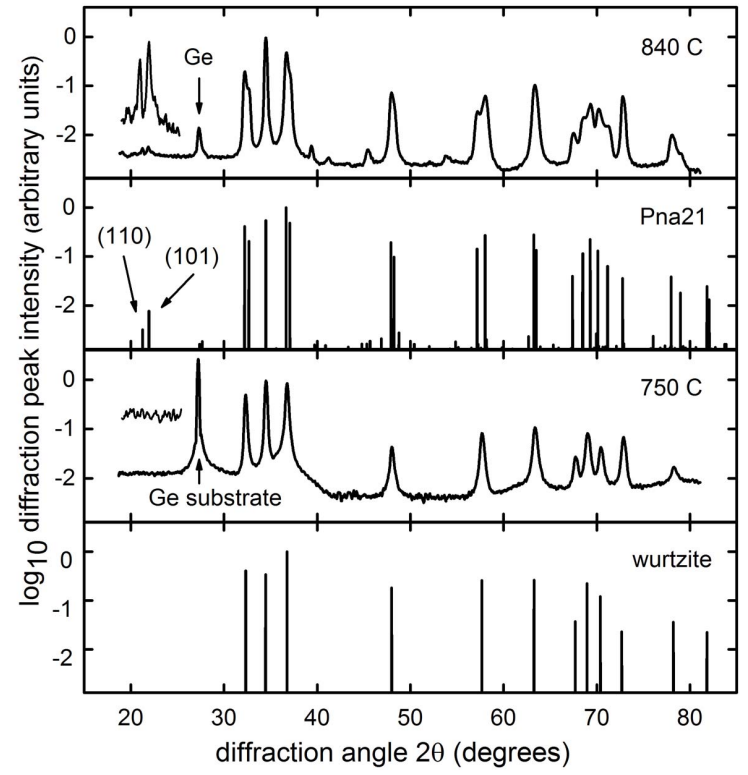

FIG. 9: Calculated and measured powder x-ray diffraction spectra of $\mathrm{ZnGeN}_{2}$. Portions of these spectra were published in Ref. 29. The insets at the left of the experimental spectra are the data for diffraction angles of $2 \theta$ between $20^{\circ}$ and $24^{\circ}$, reproduced with an expanded linear vertical scale to show better the superstructure peaks that appear for the sample grown at $840{ }^{\circ} \mathrm{C}$. These superstructure peaks are absent for the sample grown at $750{ }^{\circ} \mathrm{C}$. The peaks in the experimental spectra at the diffraction angle of approximately $27^{\circ}$ are from the Ge substrate.

and energies of formation, while structures that violate charge neutrality do not. These results justify modeling the $\mathrm{ZnSnN}_{2}$ x-ray diffraction spectrum with approximately equal proportions, and random stacking, of the two phases. This model of the lattice disorder resolves the issue between the apparent lattice disorder inferred from x-ray diffraction and inelastic light scattering measurements, and the apparent insensitivity of the band gap to the disorder. In $\mathrm{ZnGeN}_{2}$, calculations reveal larger differences between the band gaps and energies of formation of the $P n a 2_{1}$ and $P m c 2_{1}$ phases. Pna $2_{1}$ ordering results at higher growth temperatures. That disordered material was obtained at lower growth temperatures is interpreted to be a result of insufficient cation mobility during the growth process rather than a thermodynamically driven order-disorder transition.

We note, parenthetically, that the octet-rulepreserving twinned structure discovered here and shown in Fig. 2(c) is highly unusual because at the twin boundaries there are no unsatisfied bonds and the bond angles are distorted only to the extent that the atomic positions 
deviate from the ideal wurtzite positions. The geometry suggests a nanowire structure; the central core of $3 \mathrm{~A}$ $(B)$ cations in the $\alpha-(\beta-)$ plane is surrounded by the ring of $9 B(A)$ cations, then $15 A(B)$ cations in the third ring, and so on. To date, there are no reports of the synthesis of ternary nitride nanowires, but it is possible, although highly speculative, that this unique twinned configuration might be observed not only in some of the ternary nitride systems, but also in ternary systems that have chalcopyrite crystal structures in the bulk but that might have wurtzite-based configurations as nanowires. Nanowires of some III-V zincblende semiconductors can be produced in either the wurtzite or the zincblende structure, or a combination of the two, by controlling growth parameters. ${ }^{45-47}$

Finally, we emphasize the point that, although specific results here pertain only to $\mathrm{ZnSnN}_{2}$ and $\mathrm{ZnGeN}_{2}$, we expect the general concept of layered disorder in heterovalent ternaries to hold a far wider range of validity. In the nitrides, the octet-rule-violating structures are so energetically costly mainly because of the higher ionicity of the nitrides. Thus, breaking charge neutrality locally costs significantly more energy than in the less ionic III-V compounds. We expect, therefore, that restricting the disorder to octet-rule-observing configurations will be most important for other II-IV-N $\mathrm{N}_{2}$ nitrides and for other ionic heterovalent alloys such as $\mathrm{LiGaO}_{2}$. The main result of our study is that in order to explain a wurtzite-like diffraction spectrum, it is not necessary to assume fully random distribution of cations on the cation sublattice. Random stacking of the two octet-rule preserving structures is sufficient to explain fully random-looking diffraction spectra. From that point of view, it appears desirable to also reconsider this concept in the zincblendebased heterovalent ternaries. In that case also, there are exactly two ordered structures that preserve the octet rule, and a similar layered pseudospin model can be constructed. ${ }^{10}$ Thus it seems worthwhile revisiting the question of ordering in those materials. Most of the evidence in the older literature on the subject is based on the absence of superlattice peaks in x-ray diffraction just as for the wurtzite case studied here. However, we have here shown that these superlattice peaks can very easily be destroyed even within our strongly restricted layer disorder model. On the other hand, we should be clear that our model does not preclude that octet-rule-violating defects will be present in small concentrations, depending on growth conditions, even in the more ionic compounds.

\section{Acknowledgments}

K.K., E.B. G.J. and P.Q. acknowledge support from NSF grants DMR-1006132, DMR-1409346, DMR0420765, and from the U.S. Department of Education grants P200A090276 and P200A120018. J.S. and K.H. acknowledge support from the Research Corporation Scialog Program. W.L. was supported by the NSF un- der grant DMR-1104595. A.P. was supported by Faculty of Science, Chiang Mai University. Calculations made use of the High Performance Computing Resource in the Core Facility for Advanced Research Computing at Case Western Reserve University.

\section{Appendix A}

We show here that the only octet-rule-preserving ternary structures $\mathrm{ABC}_{2}$ derived from the wurtzite lattice are either sequences of the pseudospin \pm 1 layers stacked along the orthorhombic $\mathbf{b}$ axis, or the twinned structure depicted in Fig. 2(e). We arrive at this conclusion with the help of five rules that follow directly from the octet rule. The underlying wurtzite lattice is shown in Fig. 11, with the basal planes containing the cations labeled $\alpha, \beta, \gamma$ and $\delta$.

The octet rule requires that each anion be bonded to two $A$ cations and two $B$ cations. Thus only two of the three cations lying in the $\alpha$-plane that are bonded to the same anion can be of type $A$, and the fourth cation, which lies in the $\beta$-plane, must be of type $\mathrm{B}$, as illustrated in Fig. 10(a).

Rule 1, illustrated in Fig. 10(b), states that the cation in the $\alpha$-plane above the common nitrogen atom shared by three cations in the $\beta$-plane must be of type $A$ if two of the cations in the $\beta$-plane are of type $B$. The proof of this rule is shown in Figs. 10(c)-(f). The only alternative to the arrangement of Fig. 10(b) is shown in Fig. 10(c). Fig. 10(d) follows by application of the octet rule. Notice the symmetry of Fig. 10(d) about the dashed line; the cation positions $m$ and $m^{\prime}$ are equivalent. The octet rule requires that one of these be a $B$ cation, as shown in Fig. 10(e). We tilt the perspective in Fig. 10(f) to show that this arrangement in the $\beta$-plane results in a violation of the octet rule in the $\gamma$-plane; the green cation is required to be both an $A$ atom and a $B$ atom.

Rule 2, not illustrated, states that all structures that fulfill the octet rule have translational symmetry, with periodicity $c$, along the c-axis. This rule follows directly from rule 1 in the following way. Fig. 10(b) illustrates that each $A$ cation in the $\alpha$-plane must share a nitrogen atom with two $B$ cations in the $\beta$-plane. Therefore, the octet rule requires that the cation in the $\gamma$-plane beneath the $A$ cation in the $\alpha$-plane must also be an $A$ cation. Since the bonding is equivalent for all cations, the cation in the $\gamma$-plane directly below any $B$ cation in the $\alpha$-plane must also be a $B$ atom.

Rule 3 states that the triplet arrangement of Fig. $10(\mathrm{~g})$ must result in the structure shown in Fig. 2(e). Each layer added to the core triplet arrangement follows uniquely by sequential applications of the octet rule and rule 1. Rule 4 states that the arrangement of Fig. 10(h) is not allowed. Application of the octet rule and rules 1 and 3 lead to the illustrated contradiction. Rule 5 states that a type $A B A B$ row, illustrated as $B A B$ in Fig. 10(i), fixes all rows parallel to this one as type $A B A B$. There 
are two possible alternatives to rule 5, as shown. Both lead to a violation of rule 3 .

We now show that the only octet-rule-preserving structures, with the exception of the six-fold twinned structure, are sequences of \pm 1 pseudospin layers. This conclusion will follow from the conclusion that all allowed configurations have alternating $A B A B$ cations along one direction in the basal plane. We start by noting that in the $P n a 2_{1}$ phase the rows of nearest-neighbor cations in a basal plane have either $A A B B$ or $A B A B$ ordering. For the $P m c 2_{1}$ phase, the ordering of nearest-neighbor cations is $A A A A, B B B B$, or $A B A B$. We now consider the sequence $B A A$ in a basal plane. There are two possible orientations, as shown in Figs. 11(a) and (b). These are equivalent by mirror symmetry. Application of rule 4 requires one or the other of the two structures shown in Figs. 11(c) and (d). Application of rule 3 to these con- figurations leads to the arrangements of Figs. 11(e) and (f). These are equivalent by rotational symmetry, so we need only consider the consequences of the arrangement of Fig. 11(d) to cover all possibilities.

It is straightforward to generate Fig. 11(g) from Fig. 11 (d) by application of the octet rule and rules 1 and 3 . The result is the horizontal parallel $A B A B$ rows in both the $\alpha$ - and $\beta$-plane that extend infinitely, as illustrated in Fig. $11(\mathrm{~g})$. By rule 5, any additional parallel rows must be $A B A B$. Extension of the initial row $B A A$, for example to $A B B B A$, as shown by the dashed line in Fig. $11(\mathrm{~h})$, fixes the atomic arrangements of all additional parallel rows (Fig. 2(i). The sequence $A A A A$ results in the $P m c 2_{1}$ structure, and the sequence $A A B B$ results in the $P n a 2_{1}$ structure. Sequences with larger periods generate polytypes, and random sequences generate disorder along one dimension.
1 J. L. Shay and J. H. Wernick, Ternary Chalcopyrite Semiconductors: Growth, Electronic Properties, and Applications (Pergamon Press, Oxford, 1975).

2 L. I. Berger and V. D. Prochukhan, Ternary Diamond-Like Semiconductors (Metallurgiya Press, Moscow, 1968).

${ }^{3}$ K. Ramanathan, M. A. Contreras, C. L. Perkins, S. Asher, F. S. Hasoon, J. Keane, D. Young, M. Romero, W. Metzger, R. Noufi, et al., Progress in Photovoltaics: Research and Applications 11, 225 (2003), ISSN 1099-159X, URL http://dx.doi.org/10.1002/pip. 494.

${ }^{4}$ I. L. Repins, B. J. Stanbery, D. L. Young, S. S. Li, W. K. Metzger, C. L. Perkins, W. N. Shafarman, M. E. Beck, L. Chen, V. K. Kapur, et al., Progress in Photovoltaics: Research and Applications 14, 25 (2006), ISSN 1099-159X, URL http://dx.doi.org/10.1002/pip.654.

5 A. Chirilă, S. Buecheler, F. Pianezzi, P. Bloesch, C. Gretener, A. R. Uhl, C. Fella, L. Kranz, J. Perrenoud, S. Seyrling, et al., Nature Mater. 10, 857 (2011).

${ }^{6}$ C. Ohmer, Melvin and R. Pandey, MRS Bull. 23, 16 (1998).

7 S. N. Rashkeev, S. Limpijumnong, and W. R. L. Lambrecht, Phys. Rev. B 59, 2737 (1999), URL http://link. aps.org/doi/10.1103/PhysRevB.59.2737.

8 S. N. Rashkeev and W. R. L. Lambrecht, Phys. Rev. B 63, 165212 (2001), URL http://link.aps.org/doi/10.1103/ PhysRevB.63.165212.

9 M. J. Buerger, Proceedings of the $\mathrm{Na}-$ tional Academy of Sciences 20, 444 (1934), http://www.pnas.org/content/20/7/444.full.pdf+html, URL http://www .pnas .org/content/20/7/444 . short.

10 S.-H. Wei, S. B. Zhang, and A. Zunger, Phys. Rev. B 59, R2478 (1999), URL http://link.aps.org/doi/10.1103/ PhysRevB.59.R2478.

11 W. R. L. Lambrecht and A. Punya, in III-Nitride Semiconductors and their Modern Devices, edited by B. Gil (Oxford University Press, 2013), pp. 519-585.

12 L. Han, K. Kash, and H. Zhao, in Proceedings of SPIE, Light-Emitting Diodes: Materials, Devices, and Applications for Solid State Lighting XVII (2014), pp. 90030W$1-5$.

13 A. Punya and W. R. L. Lambrecht, Phys. Rev. B 88,
075302 (2013), URL http://link.aps.org/doi/10.1103/ PhysRevB. 88.075302.

14 N. Feldberg, B. Keen, J. D. Aldous, D. Scanlon, P. A. Stampe, R. Kennedy, R. Reeves, T. D. Veal, and S. Durbin, in Photovoltaic Specialists Conference (PVSC), 2012 38th IEEE (2012), pp. 002524-002527, ISSN 0160-8371.

15 N. Feldberg, J. D. Aldous, W. M. Linhart, L. J. Phillips, K. Durose, P. A. Stampe, R. J. Kennedy, D. O. Scanlon, G. Vardar, R. L. Field, et al., Applied Physics Letters 103, 042109 (2013), URL http://scitation.aip.org/ content/aip/journal/apl/103/4/10.1063/1.4816438.

16 N. C. Coronel, L. Lahourcade, K. T. Delaney, A. M. Shing, and H. A. Atwater, in Proceedings of the Photovoltaics Specialists Conference, 38th IEEE (2012), p. 003204.

17 L. Lahourcade, N. C. Coronel, K. T. Delaney, S. K. Shukla, N. A. Spaldin, and H. A. Atwater, Advanced Materials pp. n/a-n/a (2013), ISSN 1521-4095, URL http://dx.doi. org/10.1002/adma. 201204718.

18 P. Narang, S. Chen, N. C. Coronel, S. Gul, J. Yano, L.-W. Wang, N. S. Lewis, and H. A. Atwater, Advanced Materials 26, 1235 (2014), ISSN 1521-4095, URL http://dx.doi. org/10.1002/adma. 201304473.

19 P. C. Quayle, K. He, J. Shan, and K. Kash, MRS Communications FirstView, 1 (2013), ISSN 21596867, URL http://journals.cambridge.org/article_ S2159685913000190.

20 S. Chen, P. Narang, H. A. Atwater, and L.-W. Wang, Advanced Materials 26, 311 (2014), ISSN 1521-4095, URL http://dx.doi.org/10.1002/adma. 201302727.

21 F. Deng, H. Cao, L. Liang, J. Li, J. Gao, H. Zhang, R. Qin, and C. Liu, Optics Letters 40, 1282 (2015).

22 M. Maunaye and J. Lang, Mater. Res. Bull 5, 793 (1970).

23 W. L. Larson, H. P. Maruska, and A. Stevenson, J. Electrochem. Soc. 121, 1683 (1974).

24 M. Wintenberger, M. Maunaye, and Y. Laurent, Mat. Res. Bull. 8, 1049 (1973).

25 M. van Schilfgaarde, T. Kotani, and S. Faleev, Phys. Rev. Lett. 96, 226402 (2006).

26 A. Punya, W. R. L. Lambrecht, and M. van Schilfgaarde, Phys. Rev. B 84, 165204 (2011), URL http://link.aps . org/doi/10.1103/PhysRevB.84.165204. 
${ }^{27}$ K. Du, C. Bekele, C. C. Hayman, J. C. Angus, P. Pirouz, and K. Kash, J. Cryst. Growth 310, 1057 (2008).

28 T. J. Peshek, T. R. Paudel, K. Kash, and W. R. L. Lambrecht, Phys. Rev. B 77, 235213 (pages 9) (2008), URL http://link.aps.org/abstract/PRB/v77/e235213.

${ }^{29}$ E. Blanton, K. He, J. Shan, and K. Kash, in Symposium E/H Photovoltaic Technologies, Devices and Systems Based on Inorganic Materials, Small Organic Molecules and Hybrids (2013), vol. 1493 of MRS Proceedings, pp. 237-242, URL http://journals . cambridge. org/article_S1946427413002352.

30 J. P. Perdew, M. Ernzerhof, and K. Burke, J. Chem. Phys. 105, 9982 (1996), URL http://link.aip.org/ link/?JCP/105/9982/1.

31 J. Heyd, G. E. Scuseria, and M. Ernzerhof, J. Chem. Phys. 118, 8207 (2003), URL http://link.aip.org/ link/?JCP/118/8207/1.

32 J. Heyd, G. E. Scuseria, and M. Ernzerhof, J. Chem. Phys. 124, 219906 (pages 1) (2006), URL http://link.aip. org/link/? JCP/124/219906/1.

${ }^{33}$ X. Gonze, J. M. Beuken, R. Caracas, F. Detraux, M. Fuchs, G. M. Rignanese, M. Sindic, L.and Verstraete, G. Zerah, and F. Jollet, Computational Materials Science 25,3, 478 (2002/11), URL http://www .abinit.org.

34 X. Gonze, Phys. Rev. B 55, 10337 (1997).

35 X. Gonze and C. Lee, Phys. Rev. B 55, 10355 (1997).

${ }^{36}$ M. Fuchs and M. Scheffler, Comput. Phys. Commun. 119, 67 (1999).

37 J. P. Perdew and Y. Wang, Phys. Rev. B 45, 13244 (1992), URL http://link.aps.org/doi/10.1103/PhysRevB. 45. 13244.
38 U. von Barth and L. Hedin, Journal of Physics C: Solid State Physics 5, 1629 (1972), URL http://stacks.iop. org/0022-3719/5/i=13/a=012.

39 P. Hohenberg and W. Kohn, Phys. Rev. 136, B864 (1964).

40 W. Kohn and L. J. Sham, Phys. Rev. 140, A1133 (1965).

41 M. Methfessel, M. van Schilfgaarde, and R. A. Casali, in Electronic Structure and Physical Properties of Solids. The Use of the LMTO Method, edited by H. Dreyssé (Berlin Springer Verlag, 2000), vol. 535 of Lecture Notes in Physics, p. 114.

42 V. S. Kopp, V. M. Kaganer, J. Schwarzkopf, F. Waidick, T. Remmele, A. Kwasniewski, and M. Schmidbauer, Acta Crystallographica Section A 68, 148 (2012), ISSN 1600-5724, URL http://dx.doi.org/10.1107/ S0108767311044874.

43 T. R. Paudel and W. R. L. Lambrecht, Phys. Rev. B 78, 115204 (pages 12) (2008), URL http://link.aps.org/ abstract/PRB/v78/e115204.

44 J. Ma, H.-X. Deng, J.-W. Luo, and S.-H. Wei, Phys. Rev. B 90, 115201 (2014), URL http://link.aps .org/doi/10. 1103/PhysRevB.90.115201.

45 V. G. Dubrovskii, APPLIED PHYSICS LETTERS 104 (2014), ISSN 0003-6951.

46 A. Mishra, L. V. Titova, T. B. Hoang, H. E. Jackson, L. M. Smith, J. M. Yarrison-Rice, Y. Kim, H. J. Joyce, Q. Gao, H. H. Tan, et al., APPLIED PHYSICS LETTERS 91 (2007), ISSN 0003-6951.

47 K. Li, H. Sun, F. Ren, K. W. Ng, T.-T. D. Tran, R. Chen, and C. J. Chang-Hasnain, Nano letters 14, 183 (2013). 


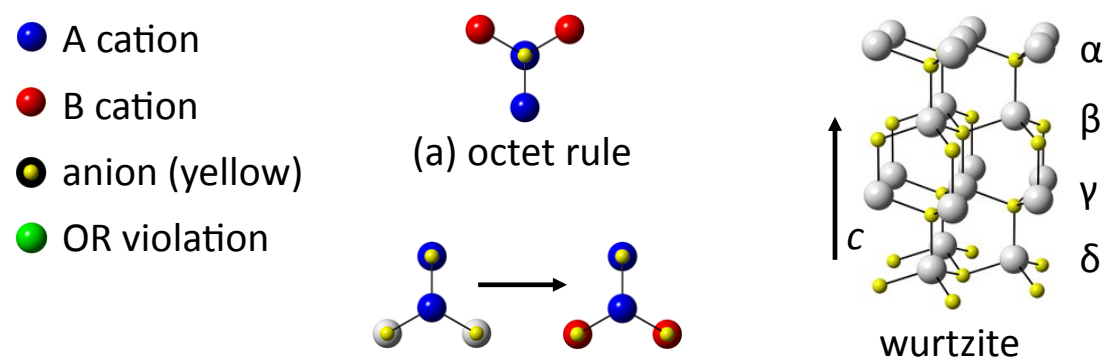

(b) rule 1
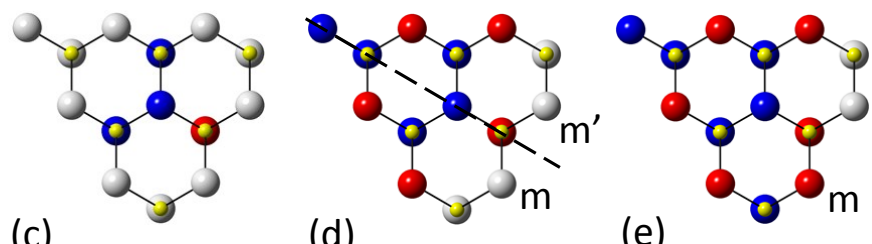

(e)
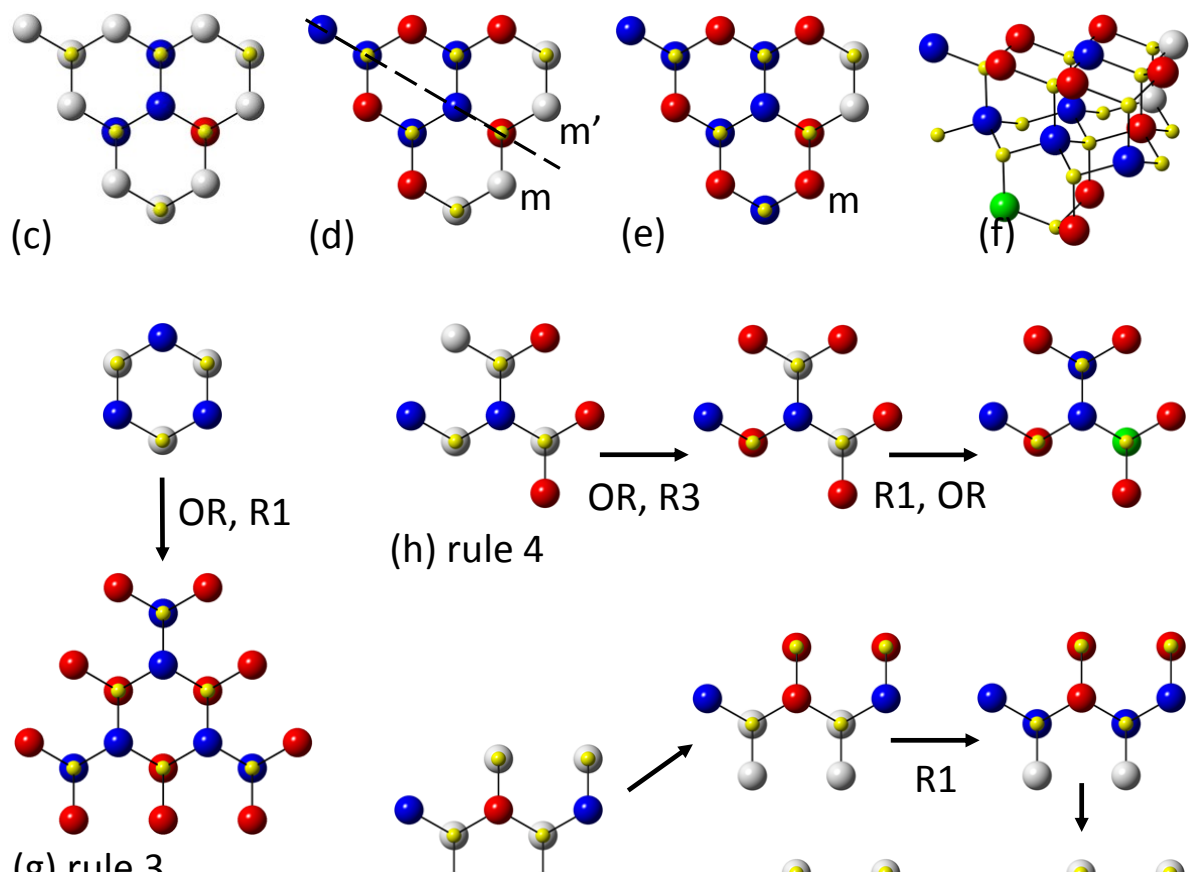

(h) rule 4

(g) rule 3

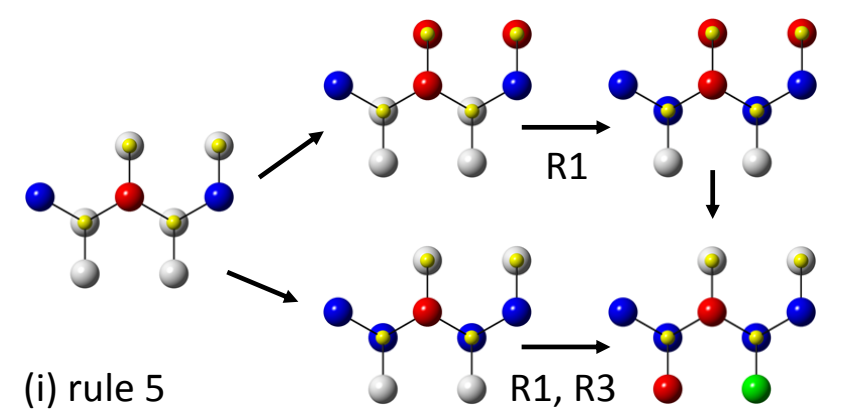

FIG. 10: Atomic arrangements associated with the rules governing the placement of A and B cations so as to preserve the octet rule.. Application of the octet rule is labeled OR, application of rule 1 is labeled R1, etc. 


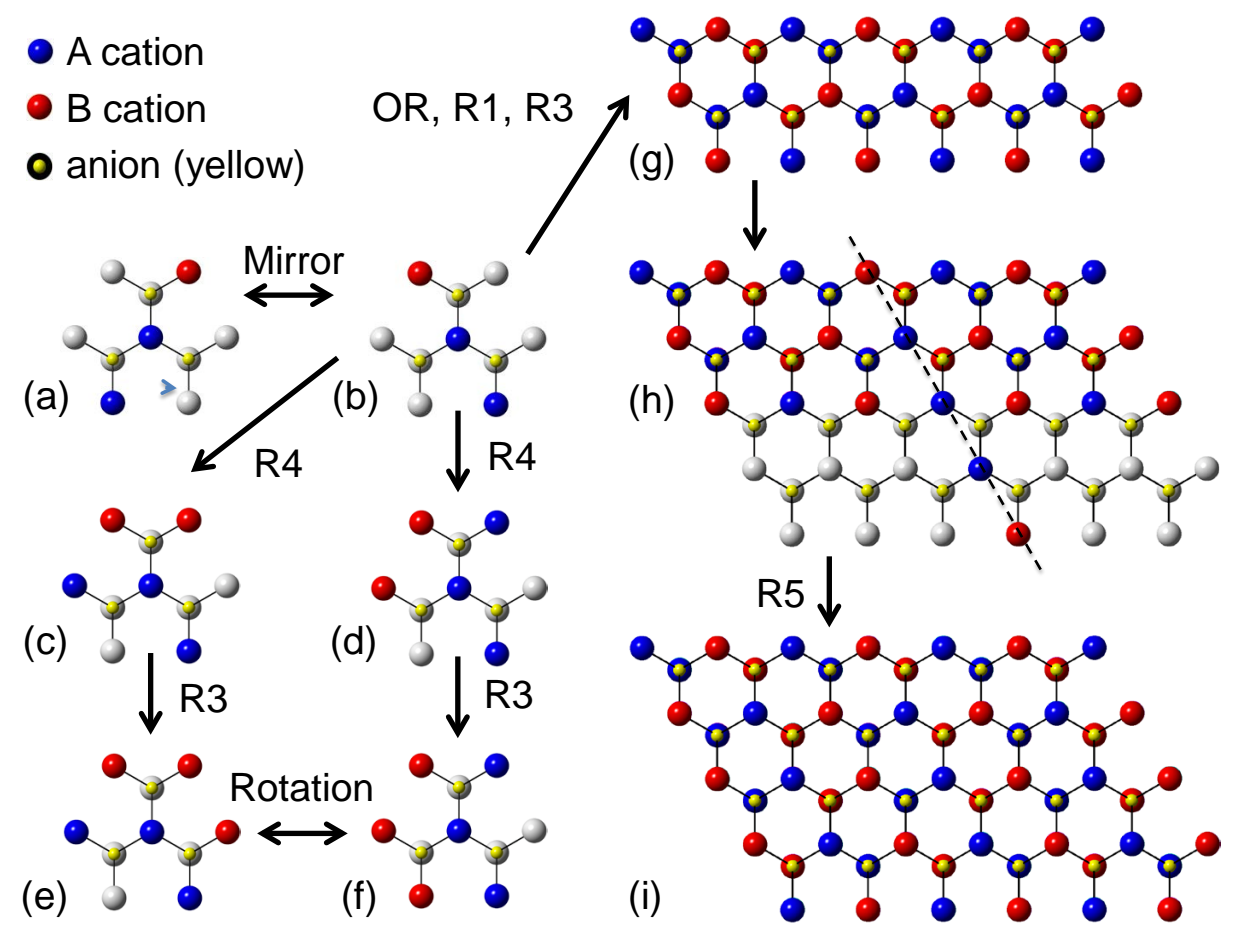

FIG. 11: Atomic arrangements associated with the proof of all possible cation placements obeying the octet rule. Application of the octet rule is labeled OR, application of rule 1 is labeled R1, etc. 